Review

\title{
A Comparative Analysis of Nanoparticle Adsorption as Fire-Protection Approach for Fabrics
}

\author{
Jenny Alongi ${ }^{1, *}$, Jennifer Tata ${ }^{1}$, Federico Carosio ${ }^{1}$, Giuseppe Rosace ${ }^{2}$, Alberto Frache ${ }^{1}$ \\ and Giovanni Camino ${ }^{1}$
}

1 Dipartimento di Scienza Applicata e Tecnologia, Politecnico di Torino, sede di Alessandria, V. Teresa Michel 5, Alessandria 15121, Italy; E-Mails: jennifertata@yahoo.com (J.T.); federico.carosio@polito.it (F.C.); alberto.frache@polito.it (A.F.); giovanni.camino@polito.it (G.C.)

2 Dipartimento di Ingegneria Industriale, Università di Bergamo, Viale Marconi 5, Dalmine (Bergamo) 24044, Italy; E-Mail: giuseppe.rosace@unibg.it

* Author to whom correspondence should be addressed; E-Mail: jenny.alongi@polito.it; Tel.: +39-013-122-9337; Fax: +39-013-122-9399.

Academic Editor: Richard A. Horrocks

Received: 24 November 2014 / Accepted: 24 December 2014 / Published: 31 December 2014

\begin{abstract}
The present paper critically analyzes the potential for commercially available nanoparticles for enhancing the flame-retardant properties of synthetic and natural fabrics and their corresponding blends. Each nanoparticle has been applied to the fabric through a finishing-like process (namely impregnation/exhausting or, more simply, nanoparticle adsorption) in aqueous media and the resulting properties of these fabrics have been assessed in terms of combustion behavior by use of a cone calorimeter under a heat flux of $35 \mathrm{~kW} / \mathrm{m}^{2}$. The influence of these nanoparticles on the main combustion parameters of polyester, cotton, and some of their blends has been thoroughly discussed. As a result of this discussion, a flame-retardant efficiency ranking of the nanoparticles under review has been established.
\end{abstract}

Keywords: nanoparticles; cotton; polyester; combustion; cone calorimeter 


\section{Introduction}

The flame retardancy of fibers and fabrics is currently one of the most important topics in both industry and academics. Indeed, recent fire statistics [1,2] point to domestic accidents involving a small flame in the presence of natural and synthetic fibers as one of the most common causes of the fatalities in domestic dwelling fires. For this reason, the standards and norms to produce flame-retardant fibers and fabrics for numerous application sectors, such as protective garments, upholstery, furniture, mattresses and bed linens, have become very strict, especially in the UK. Within this scenario, common industrial and academic practices often rely on flammability tests, such as limiting oxygen index (LOI) or vertical/horizontal flame spread tests, as useful methods to completely describe how a fabric may behave in a realistic fire scenario. These tests are not completely reliable, however, and as already shown in the literature [3-5], these tests are only able to describe the ignitability of a material (both textile and plastic) when subjected to a direct small flame application; furthermore, they do not provide any additional helpful information about the combustion behavior of the same material when irradiated by the heat flux developed by flame. To this aim, more sophisticated instrumentation like the oxygen consumption cone calorimeter can be considered a very useful tool [6,7]. Sometimes a commercial product sold as being made of a flame-retardant material can have surprising results in these flammability tests, as they are not efficient in delaying or blocking the combustion of a polymer under a certain heat flux $[8,9]$. In fact, the LOI values and combustion data collected by cone calorimetry are often not in agreement. In the past, high LOI values have been accepted as an indication of flame retardancy for a product; at present, however, this standard is not yet sufficient in order to guarantee a high safety level and has never been used as an official test standard for textiles. Indeed, the new standards for transportation, automotive, furniture and protective garments require high performances of the fabrics to the radiating panel or cone calorimetry, in addition to high LOI values [10-12].

Together with the optimization of the characterization techniques available to describe a realistic fire scenario, great attention has also been focused recently on the chemical nature of the employed flame retardant. As it is well known, the need to find halogen-free additives for synthetic fibers is becoming urgent, as well as the necessity to replace formaldehyde-based additives for cotton $[13,14]$. In this contest, flame retardants containing phosphorus compounds seem to be the most promising and suitable, although their efficiency is low if compared with that of halogens and their derivatives. On the other hand, some of the latter have been found to be toxic, bio-accumulative, and even carcinogenic [15]. Over the last few years, the use of nanoparticles in the flame-retardancy field has shown encouraging results and seems to be a valid alternative, in particular when applied as coatings on fabric surfaces [16-18]. In addition, the low amount of nanoparticles normally employed $(<1-2 \mathrm{wt} \%)$ can be seen as a great advantage. Nevertheless, not all the nanoparticles available in the market are able to act as flame retardants for all substrates. Their chemical nature plays a key role in the combustion behavior of the polymer. Furthermore, the procedure by which the nanoparticles can be deposited onto textile fibers or fabrics must be considered fundamental and pivotal for a possible industrial application. With this purpose, different approaches can be used: indeed, nanoparticles can be directly synthesized in situ on the fibers and fabric surfaces through techniques such as sol-gel processes, or alternatively, preformed nanoparticles can be deposited on the fabric surface by using new approaches like Layer-by-Layer (LbL) assembly or nanoparticle adsorption $[14,18,19]$. In the 
latter case, both natural and synthetic fabrics are initially impregnated with stable aqueous suspensions of nanoparticles and subsequently thermally treated; this process mimicking the typical impregnation/exhaustion procedure currently employed in the wet processing textile industries for conferring numerous properties to fabrics.

The present work compares the use of nanoparticles bearing different chemical structures and aspect ratios as potential flame retardants for polyester, cotton and two of their blends. The flame-retardant properties achieved have been investigated by cone calorimetry. Particular attention has been paid to the application procedure, and the use of dispersing agents or binders has been taken into consideration in order to increase the nanoparticle dispersion level, as well.

This analytical comparison deals with new results as well as preliminary data already published $[9,18]$ and collected only by the authors of the manuscript.

\section{Experimental Section}

\subsection{Materials}

Polyester $\left(\right.$ PET, area density $\left.=171 \mathrm{~g} / \mathrm{m}^{2}\right)$, cotton (area density $=212 \mathrm{~g} / \mathrm{m}^{2}$ ) and two of their blends (PET:cotton $=85: 15$ and PET:cotton $=65: 35$, area density $=280$ and $245 \mathrm{~g} / \mathrm{m}^{2}$, respectively) were kindly supplied by Klopman International (Frosinone, Italy) and used as received. A PET with higher density (area density $=490 \mathrm{~g} / \mathrm{m}^{2}$ ) kindly provided by Antecuir SA (Alicante, Spain) was used as comparison for PET with lower area density of $171 \mathrm{~g} / \mathrm{m}^{2}$.

A wide set of nanoparticles was tested as possible flame retardants; their characteristics are listed in Table 1. In order to improve their adhesion to the fabric, some dispersing agents and binders were also employed. Tables 2 and 3 summarize their chemical formulas and characteristics.

\subsection{Fabric Treatments}

Fabrics $\left(100 \times 100 \times 0.5 \mathrm{~mm}^{3}\right)$ were immersed for either 30 or $60 \mathrm{~min}$ in an aqueous nanoparticle suspension at known concentration (reported in Table 1), depending on nanoparticle dispersibility in aqueous medium. After the impregnation, the fabrics were initially dried at room temperature and subsequently placed in a compression molding machine ( 2.5 ton of pressure) at $200{ }^{\circ} \mathrm{C}$ for $10 \mathrm{~min}$ in order to fix the particles onto textile fibers.

When a dispersing agent was used, PES (dicarboxylate ester, Table 1), NSI or WP (sodium polysulfonatenaphthalene or sodium polyaminophosphonate, Table 2) was added in $0.3 \mathrm{wt} \%$ water suspension of nanoparticle for $1 \mathrm{~h}$ and mixed for $30 \mathrm{~min}$ before fabric impregnation. Subsequently, fabrics were treated following the procedure described above. The concentrations of the employed dispersing agents are detailed in Tables 4-6. More specifically, the nominal concentration ratios between ISO 5659.

B500 and $\mathrm{CNa}$ (sodium cloisite) were set at 1:4, 1:2 and 1:1 (Table 4) while that for NSI:HT (hydrotalcite), WP:HT, NSI:SiO $2, \mathrm{WP}: \mathrm{SiO}_{2}$ (Table 5) and NSI:CNa (Table 6) was set at 1:1.

Furthermore, polyester and cotton were also treated with some binders (Table 3) in order to covalently link HT, bohemite, sodium cloisite and octapropylammonium polyhedral oligomeric silsesquioxane (POSS). More specifically, the nominal nanoparticle amount was set at $1 \%$ owf (on 
weight of fabric) for all samples. The binder concentration was set at $1 \%$ owf for PES, A, B, C, and E, while 3\% owf for D binder as recommended by Europizzi s.p.a. (Bergamo, Italy) for the one-pass conventional continuous process.

\subsection{Combustion Characterization}

Cone calorimetry (fire testing technology, FTT) was employed to investigate the combustion behavior of square samples $\left(100 \times 100 \times 0.5 \mathrm{~mm}^{3}\right)$ under an irradiative heat flux of $35 \mathrm{~kW} / \mathrm{m}^{2}$ in a horizontal configuration, following the procedure described elsewhere [18]. The fabrics were placed in a sample holder and maintained in the correct configuration by a metallic grid. Time to ignition (TTI, s), total heat release (THR, $\mathrm{kW} / \mathrm{m}^{2}$ ), effective heat of combustion ( $\mathrm{EHC}, \mathrm{MJ} / \mathrm{kg}$ ), heat release rate $\left(\mathrm{HRR}, \mathrm{kW} / \mathrm{m}^{2}\right.$ ) and the corresponding peak value (pkHRR, $\mathrm{kW} / \mathrm{m}^{2}$ ) were measured. The fire performance index (FPI, $\mathrm{sm}^{2} / \mathrm{kW}$ ) was also calculated as TTI to pkHRR ratio. This last parameter was calculated in order to establish a ranking of the most efficient nanoparticles for improving the flame retardancy of polyester, cotton and their blends. Indeed, it is claimed by [20] that the higher the FPI, the better the flame-retardant performances. Furthermore, total smoke release (TSR, $\mathrm{m}^{2} / \mathrm{m}^{2}$ ), specific extinction area (SEA, $\mathrm{m}^{2} / \mathrm{kg}$ ), $\mathrm{CO}$ and $\mathrm{CO}_{2}$ yields ([CO] and $\left[\mathrm{CO}_{2}\right], \mathrm{kg} / \mathrm{kg}$ ) were evaluated, as well. In order to ensure reproducible and significant data, for each sample, the tests were repeated four times and standard deviation was calculated and added to each parameter value in Tables 4-12 which list the data collected for all experimental fabric samples studied in this current work. All fabrics were conditioned in a climatic chamber at $23 \pm 1{ }^{\circ} \mathrm{C} 50 \%$ R.H. (relative humidity) for at least $24 \mathrm{~h}$ before the combustion tests.

\section{Results and Discussion}

In order to better highlight the correlation between nanoparticle chemical structure and aspect ratio and the resulting flame-retardant properties imparted to fabrics, the discussion about the data collected by cone calorimetry will be reported in the following headings on the basis of shape factors through which nanoparticle families are classified in Table 1.

\subsection{Lamellar Nanoparticles}

\subsubsection{Montmorillonites}

Polyester fabrics: as far as montmorillonites are considered, sodium cloisite $(\mathrm{CNa})$ appears to be responsible for a significant TTI increase (192 vs. $160 \mathrm{~s}$, respectively for PET + CNa vs. PET, Table 4) and respective EHC reduction (17.5 vs. $21.8 \mathrm{MJ} / \mathrm{kg}$ ) for polyester with a consequent decrease of the burning kinetics in terms of pkHRR (80vs. $\left.90 \mathrm{~kW} / \mathrm{m}^{2}\right)$ [21] (and FPI). These parameters are plotted in Figure 1, where, in addition, the effect of the other nanoparticles is described.

This finding suggests that PET combustion mechanism is partially changed; indeed, the nanoclay acting as an insulating physical barrier somehow protects polyester from heat and oxygen during combustion; therefore, the ignition time is delayed and effective heat is reduced (a lower amount of polymer burns) (Figure 1). Furthermore, the presence of such clay on fabric surface causes a slight decrease of the TSR ( $\left.87 v s .90 \mathrm{~m}^{2} / \mathrm{m}^{2}\right)$ and of their optical density (531 vs. $\left.610 \mathrm{~m}^{2} / \mathrm{kg}, \mathrm{SEA}\right)$ as well as the $\mathrm{CO}$ and $\mathrm{CO}_{2}$ yields. 


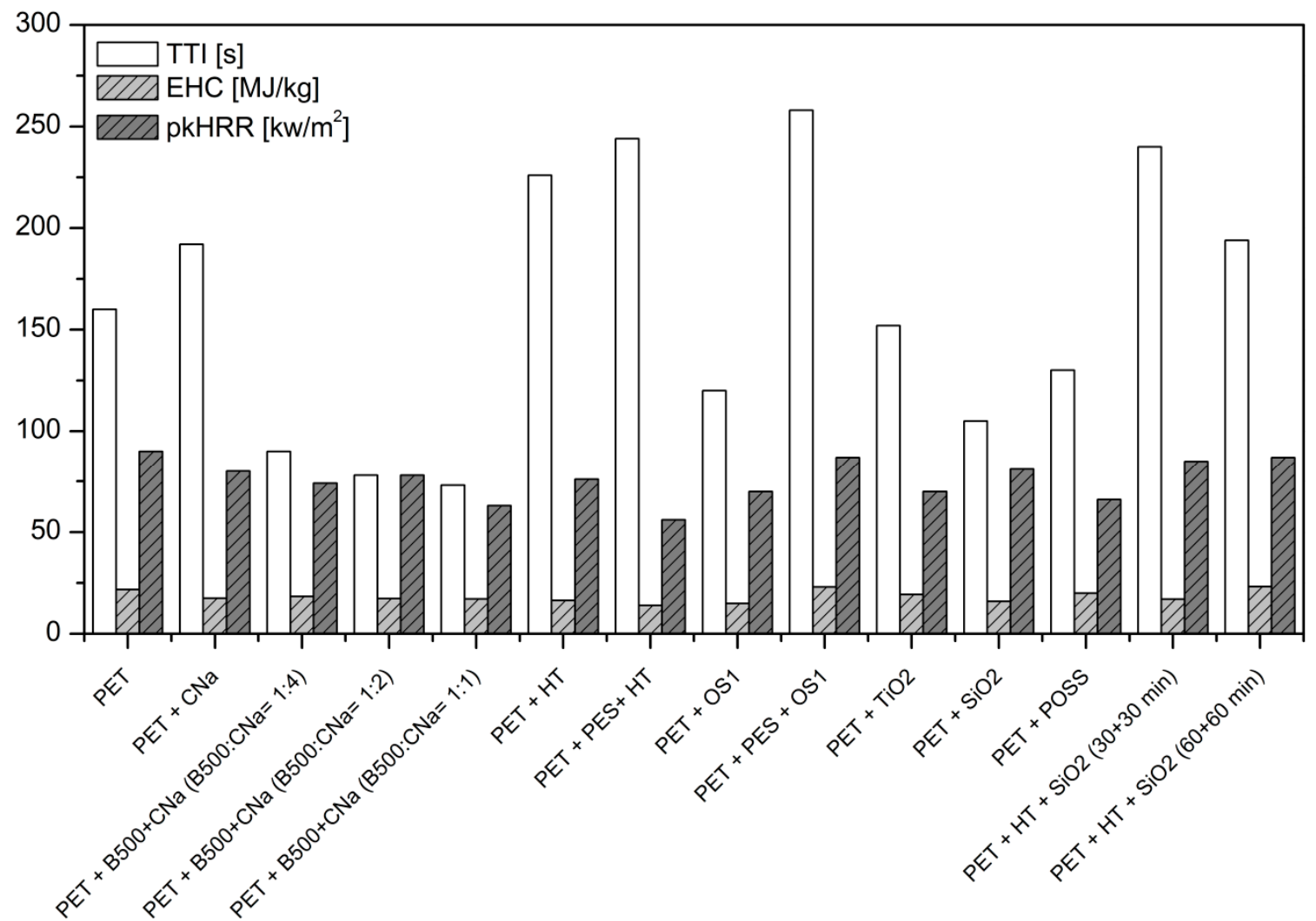

Figure 1. TTI, EHC and pkHRR values for nanoparticle-treated PET (area density $=171 \mathrm{~g} / \mathrm{m}^{2}$ ).

Table 1. Nanoparticles reviewed in this paper.

\begin{tabular}{|c|c|c|c|c|c|}
\hline Code & Family & Nanoparticle & Chemical formula & Modifier & Producer \\
\hline \multicolumn{6}{|c|}{ Lamellar nanoparticles } \\
\hline $\mathrm{CNa}$ & Montmorillonite & Sodium cloisite & $\mathrm{M}_{x}\left[\mathrm{Al}_{4-x} \mathrm{Mg}_{x}\right](\mathrm{Si})_{8} \mathrm{O}_{20}(\mathrm{OH})_{4}$ & - & $\begin{array}{l}\text { Southern Clay } \\
\text { Products, Inc. } \\
\text { (Gonzales, TX, } \\
\text { USA) }\end{array}$ \\
\hline HT & Hydrotalcite & $\begin{array}{l}\text { Carbonate } \\
\text { hydrotalcite }\end{array}$ & $\mathrm{Mg}_{6} \mathrm{Al}_{2}\left(\mathrm{CO}_{3}\right)\left(\mathrm{OH}_{16}\right) \cdot 4\left(\mathrm{H}_{2} \mathrm{O}\right)$ & Carbonate salt & $\begin{array}{l}\text { Sasol Germany } \\
\text { Gmbh (Hamburg, } \\
\text { Germany) }\end{array}$ \\
\hline OS1 & Bohemite & Sulfonate bohemite & $\mathrm{AlO}(\mathrm{OH})$ & $\begin{array}{l}p \text {-Toluenesulfonate } \\
\text { salt }\end{array}$ & $\begin{array}{l}\text { Sasol Germany } \\
\text { Gmbh (Hamburg, } \\
\text { Germany) }\end{array}$ \\
\hline \multicolumn{6}{|c|}{ Globular nanoparticles } \\
\hline $\mathrm{TiO}_{2}$ & $\begin{array}{l}\text { Titania (anatase } \\
\text { crystalline form) }\end{array}$ & Titania & $\mathrm{TiO}_{2}$ & - & $\begin{array}{l}\text { Huntsman } \\
\text { International LLC } \\
\text { (Varese, Italy) } \\
\end{array}$ \\
\hline $\mathrm{SiO}_{2}$ & Silica & Silica & $\mathrm{SiO}_{2}$ & - & $\begin{array}{l}\text { Elkem SA } \\
\text { (Dusseldorf, } \\
\text { Germany) }\end{array}$ \\
\hline POSS & $\begin{array}{l}\text { Polyhedral } \\
\text { oligomeric } \\
\text { silsesquioxane }\end{array}$ & $\begin{array}{l}\text { Octapropylammoniu } \\
\text { m POSS }^{\circledR}\end{array}$ & $\mathrm{R}(\mathrm{SiOx})$ & - & $\begin{array}{l}\text { Hybrid Plastics, } \\
\text { Inc. (Hattiesburg, } \\
\text { MS, USA) }\end{array}$ \\
\hline
\end{tabular}


Table 2. Dispersing agents.

\begin{tabular}{|c|c|c|c|}
\hline Code & Dispersing Agent & Chemical Formula & Producer \\
\hline B500 & Sodium polyacrylate & & $\begin{array}{l}\text { Europizzi s.p.a. } \\
\text { (Bergamo, Italy) }\end{array}$ \\
\hline NSI & $\begin{array}{c}\text { Sodium } \\
\text { polysulfonatenaphthalene }\end{array}$ & & $\begin{array}{l}\text { Europizzi s.p.a. } \\
\text { (Bergamo, Italy) }\end{array}$ \\
\hline \multirow[t]{2}{*}{ WP } & $\begin{array}{c}\text { Sodium } \\
\text { polyaminophosphonate }\end{array}$ & $\mathrm{Na}^{+} \mathrm{O}^{-}$ & $\begin{array}{l}\text { Bozzetto Group s.p.a. } \\
\text { (Bergamo, Italy) }\end{array}$ \\
\hline & & $\mathrm{R}=$ alkyl chain & \\
\hline
\end{tabular}

Table 3. Binders.

\begin{tabular}{|c|c|c|c|}
\hline Code & Binder & Molecular Formula & Producer \\
\hline PES & Dicarboxylate ester & OR & $\begin{array}{l}\text { Bozzetto Group s.p.a. } \\
\text { (Bergamo, Italy) }\end{array}$ \\
\hline A & Bifunctional isocyanates & & $\begin{array}{l}\text { Prochimica Group } \\
\text { (Novara, Italy) }\end{array}$ \\
\hline B & Bifunctional isocyanates & & $\begin{array}{l}\text { Prochimica Group } \\
\text { (Novara, Italy) }\end{array}$ \\
\hline $\mathrm{C}$ & Dicyanamide-formaldehyde polymer & & $\begin{array}{l}\text { Europizzi s.p.a. } \\
\text { (Bergamo, Italy) }\end{array}$ \\
\hline $\mathrm{D}$ & Melamine-formaldehyde & & $\begin{array}{l}\text { Europizzi s.p.a. } \\
\text { (Bergamo, Italy) }\end{array}$ \\
\hline $\mathrm{E}$ & Dimethylol(dihydroxy)ethyleneurea & & $\begin{array}{l}\text { Bozzetto Group s.p.a. } \\
\text { (Bergamo, Italy) }\end{array}$ \\
\hline
\end{tabular}


Table 4. Combustion data of untreated and nanoparticle-treated PET $\left(d=171 \mathrm{~g} / \mathrm{m}^{2}\right)$ by cone calorimetry.

\begin{tabular}{|c|c|c|c|c|c|c|c|c|c|}
\hline Sample & TTI (s) & THR $\left(\mathbf{M J} / \mathbf{m}^{2}\right)$ & $\begin{array}{c}\text { EHC } \\
(\mathrm{MJ} / \mathrm{kg})\end{array}$ & $\begin{array}{c}\text { pkHRR } \\
\left(\mathrm{kW} / \mathrm{m}^{2}\right)\end{array}$ & FPI $\left(\mathrm{sm}^{2} / \mathbf{k W}\right)$ & $\begin{array}{c}\text { TSR } \\
\left(\mathrm{m}^{2} / \mathrm{m}^{2}\right)\end{array}$ & $\operatorname{SEA}\left(\mathbf{m}^{2} / \mathbf{k g}\right)$ & $\begin{array}{c}{[\mathrm{CO}] \text { yield }} \\
(\mathrm{kg} / \mathrm{kg})\end{array}$ & $\begin{array}{c}{\left[\mathrm{CO}_{2}\right] \text { yield }} \\
(\mathrm{kg} / \mathrm{kg})\end{array}$ \\
\hline PET & $160 \pm 4$ & $2.5 \pm 0.6$ & $21.8 \pm 7.8$ & $90 \pm 9$ & $1.89 \pm 0.05$ & $90 \pm 8$ & $610 \pm 13$ & $0.0486 \pm 0.0228$ & $3.85 \pm 1.68$ \\
\hline \multicolumn{10}{|l|}{ Lamellar nanoparticles } \\
\hline $\mathrm{PET}+\mathrm{CNa}^{\mathrm{a}}$ & $192 \pm 7$ & $2.8 \pm 0.4$ & $17.5 \pm 2.5$ & $80 \pm 2$ & $2.35 \pm 0.04$ & $87 \pm 3$ & $531 \pm 41$ & $0.0409 \pm 0.0008$ & $3.22 \pm 0.14$ \\
\hline $\mathrm{PET}+\mathrm{B} 500+\mathrm{CNa}(\mathrm{B} 500: \mathrm{CNa}=1: 4)$ & $90 \pm 6$ & $2.5 \pm 0.1$ & $18.4 \pm 2.0$ & $74 \pm 8$ & $1.22 \pm 0.09$ & $80 \pm 6$ & $514 \pm 70$ & $0.0316 \pm 0.0049$ & $2.17 \pm 0.03$ \\
\hline $\mathrm{PET}+\mathrm{B} 500+\mathrm{CNa}(\mathrm{B} 500: \mathrm{CNa}=1: 2)$ & $78 \pm 10$ & $2.5 \pm 0.2$ & $17.3 \pm 1.8$ & $78 \pm 10$ & $0.99 \pm 0.13$ & $78 \pm 3$ & $511 \pm 34$ & $0.0305 \pm 0.0053$ & $2.03 \pm 0.24$ \\
\hline $\mathrm{PET}+\mathrm{B} 500+\mathrm{CNa}(\mathrm{B} 500: \mathrm{CNa}=1: 1)$ & $73 \pm 12$ & $2.3 \pm 0.1$ & $17.1 \pm 1.6$ & $63 \pm 2$ & $1.24 \pm 0.09$ & $72 \pm 2$ & $476 \pm 34$ & $0.0277 \pm 0.0043$ & $2.24 \pm 0.31$ \\
\hline $\mathrm{PET}+\mathrm{HT}^{\mathrm{b}}$ & $226 \pm 4$ & $2.5 \pm 0.9$ & $16.4 \pm 1.5$ & $76 \pm 7$ & $2.97 \pm 0.05$ & $82 \pm 7$ & $358 \pm 11$ & $0.0261 \pm 0.0011$ & $2.43 \pm 0.07$ \\
\hline PET + PES + HT (PES:HT = 1:1) & $244 \pm 9$ & $2.3 \pm 0.1$ & $14.0 \pm 3.1$ & $56 \pm 2$ & $4.36 \pm 0.04$ & $89 \pm 4$ & $369 \pm 36$ & $0.0227 \pm 0.0008$ & $2.45 \pm 0.19$ \\
\hline PET + OS1 & $120 \pm 6$ & $2.4 \pm 0.2$ & $15.0 \pm 0.9$ & $70 \pm 6$ & $1.50 \pm 0.06$ & $76 \pm 2$ & $394 \pm 14$ & $0.0301 \pm 0.0014$ & $2.73 \pm 0.06$ \\
\hline PET + PES + OS1 (PES:OS1 = 1:1) & $258 \pm 4$ & $2.3 \pm 0$ & $23.0 \pm 4.4$ & $87 \pm 7$ & $2.97 \pm 0.05$ & $112 \pm 9$ & $554 \pm 80$ & $0.0305 \pm 0.0046$ & $3.34 \pm 0.60$ \\
\hline \multicolumn{10}{|l|}{ Globular nanoparticles } \\
\hline $\mathrm{PET}+\mathrm{TiO}_{2}^{\mathrm{b}}$ & $152 \pm 6$ & $2.3 \pm 0.5$ & $19.4 \pm 5.0$ & $70 \pm 1$ & $2.17 \pm 0.33$ & $76 \pm 0$ & $586 \pm 17$ & $0.0421 \pm 0.0170$ & $3.58 \pm 1.19$ \\
\hline $\mathrm{PET}+\mathrm{SiO}_{2}{ }^{\mathrm{b}}$ & $105 \pm 9$ & $2.7 \pm 0.1$ & $16.0 \pm 0.2$ & $81 \pm 11$ & $1.30 \pm 0.11$ & $85 \pm 0$ & $477 \pm 11$ & $0.0302 \pm 0.0006$ & $2.71 \pm 0.12$ \\
\hline PET + POSS & $130 \pm 9$ & $2.4 \pm 0$ & $20.0 \pm 6.3$ & $66 \pm 3$ & $1.97 \pm 0.06$ & $91 \pm 0$ & $620 \pm 17$ & $0.0415 \pm 0.0158$ & $3.04 \pm 0.93$ \\
\hline \multicolumn{10}{|l|}{ Lamellar + globular nanoparticles } \\
\hline $\mathrm{PET}+\mathrm{HT}+\mathrm{SiO}_{2}(30+30 \mathrm{~min})^{\mathrm{b}}$ & $240 \pm 8$ & $3.1 \pm 0.9$ & $17.02 \pm 2.0$ & $85 \pm 8$ & $2.82 \pm 0.06$ & $95 \pm 5$ & $512 \pm 25$ & $0.0123 \pm 0.0006$ & $2.81 \pm 0.14$ \\
\hline $\mathrm{PET}+\mathrm{HT}+\mathrm{SiO}_{2}(60+60 \mathrm{~min}){ }^{\mathrm{b}}$ & $294 \pm 9$ & $2.8 \pm 0.7$ & $23.18 \pm 2.0$ & $87 \pm 9$ & $3.38 \pm 0.07$ & $92 \pm 5$ & $558 \pm 28$ & $0.0130 \pm 0.0006$ & $3.54 \pm 0.18$ \\
\hline
\end{tabular}

a PET fabrics were immersed into a $0.3 \mathrm{wt} \%$ water suspension of swollen sodium cloisite for $1 \mathrm{~h}$ and, then, fixed by thermal treatment using a laboratory; compression molding press (2.5 $\mathrm{MPa}$ of pressure and $200{ }^{\circ} \mathrm{C}$ for $10 \mathrm{~min}$ ); ${ }^{\mathrm{b}}$ data from ref. [9]. 
Table 5. Combustion data of untreated and nanoparticle-treated PET $\left(d=490 \mathrm{~g} / \mathrm{m}^{2}\right)$ by cone calorimetry.

\begin{tabular}{|c|c|c|c|c|c|c|c|c|c|}
\hline Sample & TTI (s) & $\begin{array}{c}\text { THR } \\
\left(\mathrm{MJ} / \mathbf{m}^{2}\right)\end{array}$ & $\begin{array}{c}\text { EHC } \\
(\mathrm{MJ} / \mathrm{kg})\end{array}$ & $\begin{array}{l}\text { pkHRR } \\
\left(\mathrm{kW} / \mathrm{m}^{2}\right)\end{array}$ & FPI $\left(\mathbf{s m}^{2} / \mathbf{k W}\right)$ & $\operatorname{TSR}\left(\mathrm{m}^{2} / \mathrm{m}^{2}\right)$ & SEA $\left(\mathrm{m}^{2} / \mathbf{k g}\right)$ & $\begin{array}{c}\text { [CO] Yield } \\
(\mathrm{kg} / \mathrm{kg})\end{array}$ & $\begin{array}{c}{\left[\mathrm{CO}_{2}\right] \text { Yield }} \\
(\mathrm{kg} / \mathrm{kg})\end{array}$ \\
\hline PET & $106 \pm 20$ & $6.7 \pm 0.1$ & $15.5 \pm 0.1$ & $210 \pm 16$ & $0.50 \pm 0.14$ & $227 \pm 12$ & $450 \pm 41$ & $0.0483 \pm 0.0021$ & $2.91 \pm 0.01$ \\
\hline \multicolumn{10}{|l|}{ Lamellar nanoparticles } \\
\hline $\mathrm{PET}+\mathrm{CNa}$ & $107 \pm 7$ & $6.8 \pm 0.1$ & $17.4 \pm 1.6$ & $237 \pm 25$ & $0.62 \pm 0.06$ & $226 \pm 4$ & $501 \pm 78$ & $0.0415 \pm 0.0023$ & $2.56 \pm 0.31$ \\
\hline $\mathrm{PET}+\mathrm{HT}$ & $148 \pm 2$ & $6.5 \pm 0.1$ & $16.7 \pm 0.1$ & $233 \pm 5$ & $0.64 \pm 0.02$ & $198 \pm 0$ & $416 \pm 19$ & $0.0383 \pm 0.0026$ & $2.50 \pm 0.08$ \\
\hline PET + PES + HT (PES:HT = 1:1) & $112 \pm 4$ & $6.2 \pm 0.1$ & $16.4 \pm 0.3$ & $208 \pm 6$ & $0.54 \pm 0.03$ & $171 \pm 2$ & $363 \pm 4$ & $0.0311 \pm 0.0019$ & $2.41 \pm 0.01$ \\
\hline PET + NSI + HT (NSI:HT = 1:1) & $143 \pm 3$ & $6.1 \pm 0.1$ & $17.7 \pm 1.9$ & $226 \pm 8$ & $0.63 \pm 0.03$ & $191 \pm 14$ & $424 \pm 29$ & $0.0484 \pm 0.0045$ & $2.73 \pm 0.24$ \\
\hline PET + WP + HT (WP:HT = 1:1) & $124 \pm 9$ & $6.9 \pm 0.4$ & $17.7 \pm 0.9$ & $183 \pm 18$ & $0.68 \pm 0.09$ & $193 \pm 11$ & $398 \pm 13$ & $0.0436 \pm 0.0047$ & $2.55 \pm 0.06$ \\
\hline \multicolumn{10}{|l|}{ Globular nanoparticles } \\
\hline $\mathrm{PET}+\mathrm{SiO}_{2}$ & $108 \pm 2$ & $7.1 \pm 0.2$ & $16.3 \pm 0.7$ & $215 \pm 8$ & $0.50 \pm 0.03$ & $243 \pm 8$ & $525 \pm 13$ & $0.0369 \pm 0.0003$ & $2.10 \pm 0.05$ \\
\hline $\mathrm{PET}+\mathrm{NSI}+\mathrm{SiO}_{2}\left(\mathrm{NSI}: \mathrm{SiO}_{2}=1: 1\right)$ & $119 \pm 6$ & $6.6 \pm 0.2$ & $16.4 \pm 0.7$ & $195 \pm 18$ & $0.61 \pm 0.07$ & $228 \pm 8$ & $493 \pm 33$ & $0.0397 \pm 0.0012$ & $2.50 \pm 0.11$ \\
\hline $\mathrm{PET}+\mathrm{WP}+\mathrm{SiO}_{2}\left(\mathrm{WP}: \mathrm{SiO}_{2}=1: 1\right)$ & $148 \pm 1$ & $6.6 \pm 0.1$ & $16.9 \pm 1.2$ & $244 \pm 1$ & $0.61 \pm 0.01$ & $230 \pm 0$ & $466 \pm 18$ & $0.0460 \pm 0.0042$ & $2.47 \pm 0.11$ \\
\hline
\end{tabular}

Table 6. Combustion data of untreated and nanoparticle-treated cotton $\left(d=210 \mathrm{~g} / \mathrm{m}^{2}\right)$ by cone calorimetry.

\begin{tabular}{|c|c|c|c|c|c|c|c|c|c|}
\hline Sample & TTI (s) & $\begin{array}{c}\text { THR } \\
\left(\mathbf{M J} / \mathbf{m}^{2}\right)\end{array}$ & $\begin{array}{c}\text { EHC } \\
(\mathrm{MJ} / \mathrm{kg})\end{array}$ & $\operatorname{pkHRR}\left(\mathbf{k W} / \mathbf{m}^{2}\right)$ & FPI $\left(\mathbf{s m}^{2} / \mathbf{k W}\right)$ & $\begin{array}{c}\text { TSR } \\
\left(\mathrm{m}^{2} / \mathrm{m}^{2}\right) \\
\end{array}$ & $\begin{array}{c}\text { SEA } \\
\left(\mathrm{m}^{2} / \mathrm{kg}\right) \\
\end{array}$ & $\begin{array}{c}\text { [CO] Yield } \\
(\mathrm{kg} / \mathrm{kg}) \\
\end{array}$ & $\begin{array}{c}{\left[\mathrm{CO}_{2}\right] \text { Yield }} \\
(\mathrm{kg} / \mathrm{kg}) \\
\end{array}$ \\
\hline Cotton & $14 \pm 1$ & $2.8 \pm 0$ & $17.0 \pm 0.9$ & $124 \pm 6$ & $0.11 \pm 0.06$ & $21 \pm 2$ & $25 \pm 5$ & $0.039 \pm 0.0042$ & $2.79 \pm 0.18$ \\
\hline \multicolumn{10}{|l|}{ Lamellar nanoparticles } \\
\hline Cotton $+\mathrm{CNa}$ & $38 \pm 3$ & $2.5 \pm 0.3$ & $18.0 \pm 5.4$ & $85 \pm 12$ & $0.45 \pm 0.11$ & $36 \pm 10$ & $16 \pm 7$ & $0.0613 \pm 0.0159$ & $2.68 \pm 0.88$ \\
\hline Cotton $+\mathrm{B} 500+\mathrm{CNa}(\mathrm{B} 500: \mathrm{CNa}=1: 4)$ & $19 \pm 2$ & $2.8 \pm 0.2$ & $14.6 \pm 0.6$ & $87 \pm 3$ & $0.22 \pm 0.07$ & $16 \pm 2$ & $17 \pm 2$ & $0.0416 \pm 0.0020$ & $2.48 \pm 0.06$ \\
\hline Cotton $+\mathrm{B} 500+\mathrm{CNa}(\mathrm{B} 500: \mathrm{CNa}=1: 2)$ & $22 \pm 6$ & $2.5 \pm 0.3$ & $14.2 \pm 6.5$ & $79 \pm 5$ & $0.28 \pm 0.17$ & $18 \pm 7$ & $27 \pm 3$ & $0.0719 \pm 0.0420$ & $3.29 \pm 1.17$ \\
\hline Cotton $+\mathrm{B} 500+\mathrm{CNa}(\mathrm{B} 500: \mathrm{CNa}=1: 1)$ & $18 \pm 9$ & $2.4 \pm 0.3$ & $14.8 \pm 2.1$ & $60 \pm 15$ & $0.30 \pm 0.38$ & $16 \pm 7$ & $28 \pm 3$ & $0.0499 \pm 0.0303$ & $2.40 \pm 0.23$ \\
\hline Cotton + NSI + CNa (NSI:CNa $=1: 1)$ & $22 \pm 10$ & $3.1 \pm 0.1$ & $15.1 \pm 1.5$ & $80 \pm 3$ & $0.14 \pm 0.05$ & $30 \pm 4$ & $92 \pm 4$ & $0.0198 \pm 0.0027$ & $2.30 \pm 0.05$ \\
\hline Cotton $+\mathrm{C}+\mathrm{CNa}(1 \%)$ & $40 \pm 1$ & $2.4 \pm 0.1$ & $15.9 \pm 0.5$ & $97 \pm 9$ & $0.41 \pm 0.06$ & $28 \pm 3$ & $12 \pm 6$ & $0.0675 \pm 0.0022$ & $2.55 \pm 0.11$ \\
\hline Cotton + D + CNa (1\%) & $24 \pm 3$ & $2.8 \pm 0.2$ & $20.6 \pm 0.5$ & $97 \pm 3$ & $0.25 \pm 0.08$ & $22 \pm 3$ & $7 \pm 2$ & $0.0445 \pm 0.0025$ & $3.09 \pm 0.10$ \\
\hline Cotton $+\mathrm{E}+\mathrm{CNa}(1 \%)$ & $40 \pm 2$ & $2.1 \pm 0.2$ & $15.7 \pm 6.5$ & $77 \pm 1$ & $0.52 \pm 0.03$ & $46 \pm 1$ & $16 \pm 2$ & $0.0943 \pm 0.0303$ & $2.42 \pm 0.72$ \\
\hline Cotton $+\mathrm{C}+\mathrm{CNa}(2 \%)$ & $19 \pm 3$ & $2.6 \pm 0$ & $14.9 \pm 2.9$ & $96 \pm 1$ & $0.20 \pm 0.08$ & $16 \pm 1$ & $12 \pm 3$ & $0.0464 \pm 0.0027$ & $2.38 \pm 0.30$ \\
\hline Cotton $+\mathrm{D}+\mathrm{CNa}(2 \%)$ & $24 \pm 8$ & $2.7 \pm 0.4$ & $17.0 \pm 3.1$ & $95 \pm 3$ & $0.25 \pm 0.18$ & $22 \pm 8$ & $14 \pm 7$ & $0.0512 \pm 0.0148$ & $2.64 \pm 0.45$ \\
\hline
\end{tabular}


Table 6. Cont.

\begin{tabular}{|c|c|c|c|c|c|c|c|c|c|}
\hline Sample & TTI (s) & $\begin{array}{c}\text { THR } \\
\left(\mathrm{MJ} / \mathbf{m}^{2}\right)\end{array}$ & $\begin{array}{c}\text { EHC } \\
(\mathrm{MJ} / \mathrm{kg})\end{array}$ & pkHRR (kW/m²) & FPI $\left(\mathbf{s m}^{2} / \mathbf{k W}\right)$ & $\begin{array}{c}\text { TSR } \\
\left(\mathrm{m}^{2} / \mathbf{m}^{2}\right)\end{array}$ & $\begin{array}{c}\text { SEA } \\
\left(\mathrm{m}^{2} / \mathbf{k g}\right) \\
\end{array}$ & $\begin{array}{c}{[\mathrm{CO}] \text { Yield }} \\
(\mathrm{kg} / \mathrm{kg})\end{array}$ & $\begin{array}{c}{\left[\mathrm{CO}_{2}\right] \text { Yield }} \\
(\mathrm{kg} / \mathrm{kg})\end{array}$ \\
\hline Cotton $+\mathrm{E}+\mathrm{CNa}(2 \%)$ & $36 \pm 3$ & $2.4 \pm 0.1$ & $18.4 \pm 4.4$ & $92 \pm 6$ & $0.39 \pm 0.07$ & $7 \pm 3$ & $10 \pm 7$ & $0.0637 \pm 0.0267$ & $2.81 \pm 0.59$ \\
\hline Cotton $+\mathrm{C}+\mathrm{CNa}(5 \%)$ & $20 \pm 4$ & $2.8 \pm 0.2$ & $14.8 \pm 1.8$ & $94 \pm 7$ & $0.13 \pm 0.02$ & $22 \pm 3$ & $12 \pm 4$ & $0.0472 \pm 0.0034$ & $2.43 \pm 0.24$ \\
\hline Cotton + D + CNa (5\%) & $32 \pm 4$ & $2.4 \pm 0.2$ & $14.6 \pm 1.5$ & $91 \pm 11$ & $0.35 \pm 0.12$ & $14 \pm 8$ & $12 \pm 7$ & $0.0575 \pm 0.0122$ & $2.41 \pm 0.33$ \\
\hline Cotton $+\mathrm{E}+\mathrm{CNa}(5 \%)$ & $22 \pm 4$ & $2.7 \pm 0.2$ & $14.7 \pm 1.5$ & $97 \pm 7$ & $0.23 \pm 0.13$ & $9 \pm 2$ & $6 \pm 2$ & $0.0578 \pm 0.0061$ & $2.36 \pm 0.18$ \\
\hline \multicolumn{10}{|l|}{ Globular nanoparticles } \\
\hline Cotton + POSS ${ }^{b}$ & $18 \pm 3$ & $2.2 \pm 0.1$ & $15.3 \pm 3.5$ & $76 \pm 2$ & $0.24 \pm 0.10$ & $9 \pm 3$ & $16 \pm 3$ & $0.0697 \pm 0.0169$ & $2.27 \pm 0.55$ \\
\hline Cotton $+C+$ POSS $(1 \%)^{b}$ & $34 \pm 1$ & $2.2 \pm 0.1$ & $14.8 \pm 1.0$ & $85 \pm 4$ & $0.40 \pm 0.04$ & $35 \pm 1$ & $16 \pm 4$ & $0.0761 \pm 0.0106$ & $2.18 \pm 0.04$ \\
\hline Cotton + D + POSS $(1 \%)^{b}$ & $20 \pm 5$ & $2.4 \pm 0.1$ & $14.0 \pm 0.6$ & $84 \pm 4$ & $0.24 \pm 0.15$ & $17 \pm 9$ & $16 \pm 4$ & $0.0595 \pm 0.0112$ & $2.25 \pm 0.09$ \\
\hline Cotton $+E+\operatorname{POSS}(1 \%)^{b}$ & $30 \pm 2$ & $2.3 \pm 0$ & $15.9 \pm 1.7$ & $93 \pm 6$ & $0.32 \pm 0.07$ & $27 \pm 0$ & $27 \pm 7$ & $0.0762 \pm 0.0051$ & $2.62 \pm 0.28$ \\
\hline Cotton $+\mathrm{C}+\operatorname{POSS}(2 \%)^{\mathrm{b}}$ & $30 \pm 4$ & $1.7 \pm 0.2$ & $13.8 \pm 0.9$ & $58 \pm 9$ & $0.52 \pm 0.14$ & $57 \pm 14$ & $15 \pm 3$ & $0.1417 \pm 0.0298$ & $2.54 \pm 0.19$ \\
\hline Cotton $+\mathrm{D}+\operatorname{POSS}(2 \%)^{\mathrm{b}}$ & $34 \pm 4$ & $1.5 \pm 0.1$ & $12.3 \pm 1.3$ & $52 \pm 5$ & $0.65 \pm 0.11$ & $57 \pm 7$ & $15 \pm 3$ & $0.1486 \pm 0.0351$ & $2.49 \pm 0.24$ \\
\hline Cotton $+E+\operatorname{POSS}(2 \%)^{b}$ & $36 \pm 2$ & $1.8 \pm 0.2$ & $14.3 \pm 1.1$ & $67 \pm 6$ & $0.54 \pm 0.07$ & $42 \pm 12$ & $14 \pm 2$ & $0.1302 \pm 0.0199$ & $2.73 \pm 0.17$ \\
\hline Cotton $+C+\operatorname{POSS}(5 \%)^{b}$ & $26 \pm 2$ & $1.9 \pm 0.1$ & $13.2 \pm 0.3$ & $62 \pm 4$ & $0.42 \pm 0.07$ & $27 \pm 1$ & $15 \pm 3$ & $0.0805 \pm 0.0063$ & $2.28 \pm 0.17$ \\
\hline Cotton + D + POSS $(5 \%)^{\mathrm{b}}$ & $30 \pm 7$ & $1.3 \pm 0.3$ & $13.9 \pm 1.6$ & $44 \pm 9$ & $0.68 \pm 0.22$ & $39 \pm 14$ & $20 \pm 4$ & $0.1150 \pm 0.0323$ & $2.54 \pm 0.16$ \\
\hline Cotton + E + POSS $(5 \%)^{b}$ & $28 \pm 3$ & $2.1 \pm 0.3$ & $13.9 \pm 1.6$ & $67 \pm 7$ & $0.42 \pm 0.11$ & $23 \pm 6$ & $26 \pm 4$ & $0.0713 \pm 0.0140$ & $2.41 \pm 0.14$ \\
\hline \multicolumn{10}{|l|}{ Lamellar + globular nanoparticles } \\
\hline Cotton $+\mathrm{C}+\mathrm{CNa}(1 \%)+\operatorname{POSS}(1 \%)$ & $30 \pm 2$ & $2.3 \pm 0.1$ & $20.0 \pm 5.1$ & $90 \pm 6$ & $0.33 \pm 0.07$ & $27 \pm 3$ & $15 \pm 6$ & $0.0875 \pm 0.0168$ & $3.18 \pm 0.83$ \\
\hline Cotton + D + CNa $(1 \%)+\operatorname{POSS}(1 \%)$ & $21 \pm 0$ & $2.5 \pm 0.1$ & $13.1 \pm 0.6$ & $102 \pm 4$ & $0.21 \pm 0.02$ & $13 \pm 2$ & $20 \pm 0$ & $0.0486 \pm 0.0096$ & $1.83 \pm 0$ \\
\hline Cotton $+\mathrm{E}+\mathrm{CNa}(1 \%)+\operatorname{POSS}(1 \%)$ & $22 \pm 5$ & $2.4 \pm 0.3$ & $13.0 \pm 2.6$ & $84 \pm 14$ & $0.26 \pm 0.20$ & $18 \pm 10$ & $18 \pm 2$ & $0.0539 \pm 0.0143$ & $1.76 \pm 0.34$ \\
\hline Cotton $+\mathrm{C}+\mathrm{CNa}(2.5 \%)+\operatorname{POSS}(2.5 \%)$ & $20 \pm 1$ & $2.2 \pm 0.1$ & $14.4 \pm 0.4$ & $93 \pm 7$ & $0.22 \pm 0.06$ & $15 \pm 5$ & $14 \pm 3$ & $0.0699 \pm 0.0002$ & $2.40 \pm 0.04$ \\
\hline Cotton + D + CNa $(2.5 \%)+\operatorname{POSS}(2.5 \%)$ & $20 \pm 6$ & $2.0 \pm 0.1$ & $12.6 \pm 0.4$ & $70 \pm 5$ & $0.29 \pm 0.19$ & $25 \pm 6$ & $17 \pm 8$ & $0.0759 \pm 0.0064$ & $2.11 \pm 0.09$ \\
\hline Cotton $+\mathrm{E}+\mathrm{CNa}(2.5 \%)+\operatorname{POSS}(2.5 \%)$ & $26 \pm 5$ & $2.0 \pm 0.1$ & $13.3 \pm 1.1$ & $70 \pm 9$ & $0.37 \pm 0.16$ & $30 \pm 8$ & $19 \pm 8$ & $0.0939 \pm 0.0266$ & $2.28 \pm 0.13$ \\
\hline Cotton $+\mathrm{HT}+\mathrm{SiO}_{2}(30+30 \mathrm{~min})^{\mathrm{a}}$ & $30 \pm 5$ & $2.9 \pm 0.3$ & $2.9 \pm 0.3$ & $93 \pm 8$ & $0.32 \pm 0.13$ & $18 \pm 10$ & $11 \pm 7$ & $0.0254 \pm 0.0067$ & $2.08 \pm 0.53$ \\
\hline Cotton $+\mathrm{HT}+\mathrm{SiO}_{2}(60+60 \mathrm{~min})^{\mathrm{a}}$ & $37 \pm 4$ & $2.6 \pm 0.1$ & $2.6 \pm 0.1$ & $86 \pm 4$ & $0.43 \pm 0.08$ & $22 \pm 7$ & $9 \pm 5$ & $0.0291 \pm 0.0053$ & $2.14 \pm 0.07$ \\
\hline
\end{tabular}

${ }^{a}$ data from ref. [22]; ${ }^{b}$ data from ref. [23]. 
In order to further increase PET-CNa interactions, a pre-treatment of the fabric by cold oxygen plasma (etching) was undertaken with different conditions of power and time (Table 7), as already demonstrated by our group in a previous paper [21]. In this case, no dispersing agent was employed to further increase the amount of nanoparticles in aqueous suspension.

The results collected by cone calorimetry and scanning electron microscopy (magnifications in ref. [21]) show how the plasma pre-treatment can increase both clay surface density and the interactions between inorganic nanoparticles and PET surface. The best sample (PET $+\mathrm{CNa} 5$ ) has exhibited an increase in time to ignition up to $104 \%$ and a reduction in the heat release rate of $10 \%$.

Table 7. Combustion data of PET $\left(d=171 \mathrm{~g} / \mathrm{m}^{2}\right)$ treated with sodium cloisite and plasma treatment by cone calorimetry [21].

\begin{tabular}{|c|c|c|c|c|c|c|}
\hline Sample & $\begin{array}{c}\text { Plasma } \\
\text { Power (w) }\end{array}$ & $\begin{array}{c}\text { Plasma } \\
\text { Time (s) }\end{array}$ & TTI (s) & THR $\left(\mathrm{MJ} / \mathrm{m}^{2}\right)$ & pkHRR $\left(\mathrm{kW} / \mathrm{m}^{2}\right)$ & FPI $\left(\mathrm{sm}^{2} / \mathrm{kW}\right)$ \\
\hline PET & - & - & $158 \pm 4$ & $2.5 \pm 0.4$ & $90 \pm 9$ & $1.76 \pm 0.06$ \\
\hline $\mathrm{PET}+\mathrm{Can}^{\mathrm{a}}$ & - & - & $198 \pm 8$ & $2.8 \pm 0.4$ & $105 \pm 2$ & $1.89 \pm 0.03$ \\
\hline $\mathrm{PET}+\mathrm{CNa}{ }^{1}{ }^{\mathrm{a}}$ & 120 & 15 & $244 \pm 24$ & $2.4 \pm 0.4$ & $85 \pm 7$ & $2.87 \pm 0.08$ \\
\hline $\mathrm{PET}+\mathrm{CNa}_{-} 2^{\mathrm{a}}$ & 120 & 60 & $261 \pm 10$ & $2.6 \pm 0.1$ & $89 \pm 7$ & $2.93 \pm 0.03$ \\
\hline $\mathrm{PET}+\mathrm{CNa}_{-} 3^{\mathrm{a}}$ & 120 & 80 & $192 \pm 34$ & $2.3 \pm 0.4$ & $94 \pm 12$ & $2.04 \pm 0.15$ \\
\hline $\mathrm{PET}+\mathrm{CNa}_{-} 4^{\mathrm{a}}$ & 50 & 180 & $241 \pm 10$ & $2.2 \pm 0.8$ & $92 \pm 19$ & $2.62 \pm 0.07$ \\
\hline $\mathrm{PET}+\mathrm{CNa} 5^{5^{\mathrm{a}}}$ & 80 & 180 & $322 \pm 29$ & $1.8 \pm 0.4$ & $81 \pm 18$ & $3.98 \pm 0.16$ \\
\hline
\end{tabular}

a $0.3 \mathrm{wt} \%$ water suspension of swollen sodium cloisite was used for these treatments.

In the opinion of the authors, the highest risk connected with the proposed approach of nanoparticle adsorption (namely, simple impregnation) can be the low swelling factor of the nanoclay within the aqueous medium; in this manner, a low amount of nanoparticles could be deposited on the fabric surface. For this reason, in the present study, the use of dispersing agents has been taken into account in order to increase the dispersion level of available sodium cloisite. To this aim, a sodium polyacrylate binding agent (B500, Table 2), commonly used in industrial fabric finishing, was employed in different concentrations (B500: $\mathrm{CNa}=1: 4,1: 2$ and 1:1, respectively) and the behavior of so-treated fabrics have been investigated (Table 4). The presence of this species strongly influences PET TTI values such that they decrease $(90,78$, and 73 s vs. 160 and 192 s for neat PET or PET $+\mathrm{CNa})$ (Figure 1). A possible explanation can be related to the low thermal stability of the employed polyacrylate. Upon exposure to the cone heat flux, the early degradation of the polyacrylate could lead to the production of an overall increased amount of combustible volatiles, thus sensitizing the ignition of the sample. In spite of these results, it is possible to observe a significant reduction of (i) the combustion kinetics parameters in terms of pkHRR (Figure 1); (ii) smoke generation (TSR and SEA) and (iii) $\mathrm{CO}$ and $\mathrm{CO}_{2}$ yields as compared with the treatment with sodium cloisite alone. It is likely that a higher amount of nanoparticles have been deposited on the fabric surface, creating a more efficient physical barrier during PET combustion. However, from an overall consideration, assessing the FPI values for these systems, it is possible to conclude that the use of the B500 dispersing agent in combination with the $\mathrm{CNa}$ does not improve the flame-retardant properties of cotton as well as when only sodium cloisite is present (Table 4). 
Table 8. Combustion data of untreated and nanoparticle-treated PET-cotton $=85: 15\left(d=280 \mathrm{~g} / \mathrm{m}^{2}\right)$ blends by cone calorimetry.

\begin{tabular}{|c|c|c|c|c|c|c|c|c|c|}
\hline Sample & TTI (s) & THR (MJ/m²) & EHC (MJ/kg) & $\begin{array}{c}\text { pkHRR } \\
\left(\mathrm{kW} / \mathrm{m}^{2}\right)\end{array}$ & $\begin{array}{c}\text { FPI } \\
\left(\mathrm{sm}^{2} / \mathbf{k W}\right)\end{array}$ & $\operatorname{TSR}\left(\mathrm{m}^{2} / \mathrm{m}^{2}\right)$ & $\begin{array}{c}\text { SEA } \\
\left(\mathrm{m}^{2} / \mathrm{kg}\right)\end{array}$ & $\begin{array}{c}\text { [CO] Yield } \\
(\mathrm{kg} / \mathrm{kg})\end{array}$ & $\begin{array}{c}{\left[\mathrm{CO}_{2}\right] \text { Yield }} \\
(\mathrm{kg} / \mathrm{kg})\end{array}$ \\
\hline PET - cotton $($ PET:cotton $=85: 15)$ & $35 \pm 5$ & $3.7 \pm 0.1$ & $15.3 \pm 0.4$ & $150 \pm 1$ & $0.24 \pm 0.07$ & $107 \pm 2$ & $407 \pm 6$ & $0.0380 \pm 0$ & $2.94 \pm 0.03$ \\
\hline \multicolumn{10}{|l|}{ Lamellar nanoparticles } \\
\hline PET-cotton $85: 15+\mathrm{CNa}$ & $52 \pm 5$ & $3.6 \pm 0.6$ & $15.8 \pm 3.4$ & $107 \pm 13$ & $0.49 \pm 0.19$ & $122 \pm 7$ & $460 \pm 26$ & $0.0370 \pm 0.0034$ & $2.62 \pm 0.20$ \\
\hline \multicolumn{10}{|l|}{ Globular nanoparticles } \\
\hline $\mathrm{PET}-$ cotton $85: 15+\mathrm{SiO}_{2}(30 \mathrm{~min})$ & $26 \pm 3$ & $4.2 \pm 0.1$ & $16.4 \pm 0.2$ & $131 \pm 8$ & $0.20 \pm 0.09$ & $109 \pm 4$ & $417 \pm 13$ & $0.0264 \pm 0.0009$ & $2.43 \pm 0.03$ \\
\hline PET-cotton $85: 15+\mathrm{SiO}_{2}(60 \mathrm{~min})$ & $56 \pm 7$ & $3.9 \pm 0.3$ & $16.2 \pm 0.5$ & $128 \pm 8$ & $0.44 \pm 0.09$ & $118 \pm 6$ & $435 \pm 34$ & $0.0316 \pm 0.0015$ & $2.55 \pm 0.07$ \\
\hline
\end{tabular}

Table 9. Combustion data of untreated and nanoparticle-treated PET-cotton $=65: 35$ blend $\left(\mathrm{d}=245 \mathrm{~g} / \mathrm{m}^{2}\right)$ by cone calorimetry.

\begin{tabular}{|c|c|c|c|c|c|c|c|c|c|}
\hline Sample & TTI (s) & $\begin{array}{c}\text { THR } \\
\left(\mathrm{MJ} / \mathbf{m}^{2}\right)\end{array}$ & $\begin{array}{c}\text { EHC } \\
(\mathrm{MJ} / \mathrm{kg})\end{array}$ & $\begin{array}{c}\text { pkHRR } \\
\left(\mathrm{kW} / \mathrm{m}^{2}\right)\end{array}$ & FPI $\left(\mathbf{s m}^{2} / \mathbf{k W}\right)$ & $\begin{array}{c}\text { TSR } \\
\left(\mathrm{m}^{2} / \mathrm{m}^{2}\right)\end{array}$ & $\begin{array}{c}\text { SEA } \\
\left(\mathrm{m}^{2} / \mathrm{kg}\right)\end{array}$ & $\begin{array}{c}\text { [CO] Yield } \\
(\mathrm{kg} / \mathrm{kg})\end{array}$ & $\begin{array}{c}{\left[\mathrm{CO}_{2}\right] \text { Yield }} \\
(\mathrm{kg} / \mathrm{kg})\end{array}$ \\
\hline PET - cotton $($ PET: cotton $=65: 35)$ & $11 \pm 1$ & $3.4 \pm 0.2$ & $15.3 \pm 0.3$ & $140 \pm 5$ & $0.08 \pm 0.06$ & $53 \pm 9$ & $259 \pm 12$ & $0.0313 \pm 0.0021$ & $2.91 \pm 0.03$ \\
\hline \multicolumn{10}{|l|}{ Lamellar nanoparticles } \\
\hline PET-cotton $65: 35+\mathrm{CNa}$ & $24 \pm 4$ & $3.7 \pm 0.1$ & $17.1 \pm 0.3$ & $125 \pm 7$ & $0.19 \pm 0.13$ & $76 \pm 1$ & $329 \pm 24$ & $0.0328 \pm 0.0049$ & $2.54 \pm 0.11$ \\
\hline PET-cotton $65: 35+$ HT $(30 \mathrm{~min})$ & $74 \pm 1$ & $2.7 \pm 0.3$ & $15.2 \pm 2.6$ & $121 \pm 1$ & $0.61 \pm 0.01$ & $102 \pm 2$ & $315 \pm 50$ & $0.0304 \pm 0.0023$ & $2.71 \pm 0.54$ \\
\hline PET-cotton $65: 35+$ HT $(60 \mathrm{~min})$ & $20 \pm 8$ & $3.0 \pm 0.2$ & $16.3 \pm 0.8$ & $94 \pm 6$ & $0.21 \pm 0.23$ & $65 \pm 9$ & $302 \pm 15$ & $0.0219 \pm 0.0015$ & $2.71 \pm 0.12$ \\
\hline \multicolumn{10}{|l|}{ Globular nanoparticles } \\
\hline PET-cotton $65: 35+\mathrm{SiO}_{2}(30 \mathrm{~min})$ & $20 \pm 1$ & $3.6 \pm 0.1$ & $16.7 \pm 1.3$ & $130 \pm 5$ & $0.15 \pm 0.04$ & $65 \pm 5$ & $289 \pm 23$ & $0.0265 \pm 0.0028$ & $2.57 \pm 0.21$ \\
\hline PET-cotton $65: 35+\mathrm{SiO}_{2}(60 \mathrm{~min})$ & $20 \pm 1$ & $3.4 \pm 0.1$ & $23.2 \pm 13.4$ & $127 \pm 2$ & $0.16 \pm 0.03$ & $65 \pm 3$ & $266 \pm 54$ & $0.0365 \pm 0.0214$ & $2.44 \pm 0.68$ \\
\hline \multicolumn{10}{|l|}{ Lamellar + globular nanoparticles } \\
\hline $\mathrm{PET}-$ cotton $+\mathrm{HT}+\mathrm{SiO}_{2}(30+30 \mathrm{~min})$ & $54 \pm 1$ & $2.6 \pm 0.3$ & $16.5 \pm 4.1$ & $115 \pm 4$ & $0.47 \pm 0.03$ & $81 \pm 1$ & $337 \pm 70$ & $0.0281 \pm 0.0041$ & $2.69 \pm 0.47$ \\
\hline $\mathrm{PET}-$ cotton $+\mathrm{HT}+\mathrm{SiO}_{2}(60+60 \mathrm{~min})$ & $40 \pm 2$ & $3.4 \pm 0.3$ & $15.8 \pm 2.0$ & $105 \pm 7$ & $0.39 \pm 0.08$ & $58 \pm 2$ & $266 \pm 38$ & $0.0076 \pm 0.0003$ & $2.51 \pm 0.41$ \\
\hline
\end{tabular}


The same nanoparticle has been tested using a PET fabric having a higher area density rather than of that previously used $\left(490 \mathrm{~g} / \mathrm{m}^{2} v s .171 \mathrm{~g} / \mathrm{m}^{2}\right)$. The data collected in Table 5 show that sodium cloisite is not effective in enhancing the flame retardancy of this heavier weight polyester. This can be ascribed to the denser structure of this fabric that inhibits nanoclay adsorption on PET surface. On the other hand, it is reasonable to conclude that doubling the area density will double the amount of fuel per unit area and that, if the level of clay is the same, it will be less efficient at reducing flammability.

Cotton: as far as cotton is concerned (Table 6), sodium cloisite shows that is able to increase its TTI in a remarkable way (38 s vs. $14 \mathrm{~s}$ for cotton $+\mathrm{CNa}$ and cotton, respectively), reducing pkHRR $\left(87 \mathrm{~kW} / \mathrm{m}^{2}\right.$ vs. $124 \mathrm{~kW} / \mathrm{m}^{2}$, a reduction of $\left.30 \%\right)$ at the same time. The resulting FPI value is higher than that of pure cotton $\left(0.45 \mathrm{sm}^{2} / \mathrm{kW} v s .0 .11 \mathrm{sm}^{2} / \mathrm{kW}\right)$.

Also in this case, the B500 chemical has been employed as dispersing agent for improving sodium cloisite dispersion level; once again, a strong decrease of cotton TTI occurs (regardless of B500:CNa ratio), but also a significant decrease of pkHRR is observed in comparison with the pure fabric, as shown in Figure 2. However, the highest reduction was assessed when the B500:CNa ratio was 1:1.

An alternative dispersing agent under study has been sodium polysulfonatenaphthalene (NSI, Table 2). This species was assumed to be more promising than the sodium polyacrylate as it should not hydrolyze cotton and, in addition, naphthalene groups could promote cellulose carbonization, favoring a somewhat flame-retardant effect. In spite of these considerations, the performances of NSI-treated fabrics have not exhibited any improvements in terms of TTI, EHC and pkHRR (Table 6) if compared with the fabric treated with only $\mathrm{CNa}$ (Figure 2).

However, it is important to highlight that $\mathrm{CNa}$ (regardless of its amount) causes a substantial increase in total smokes and $\mathrm{CO}$ and $\mathrm{CO}_{2}$ yields.

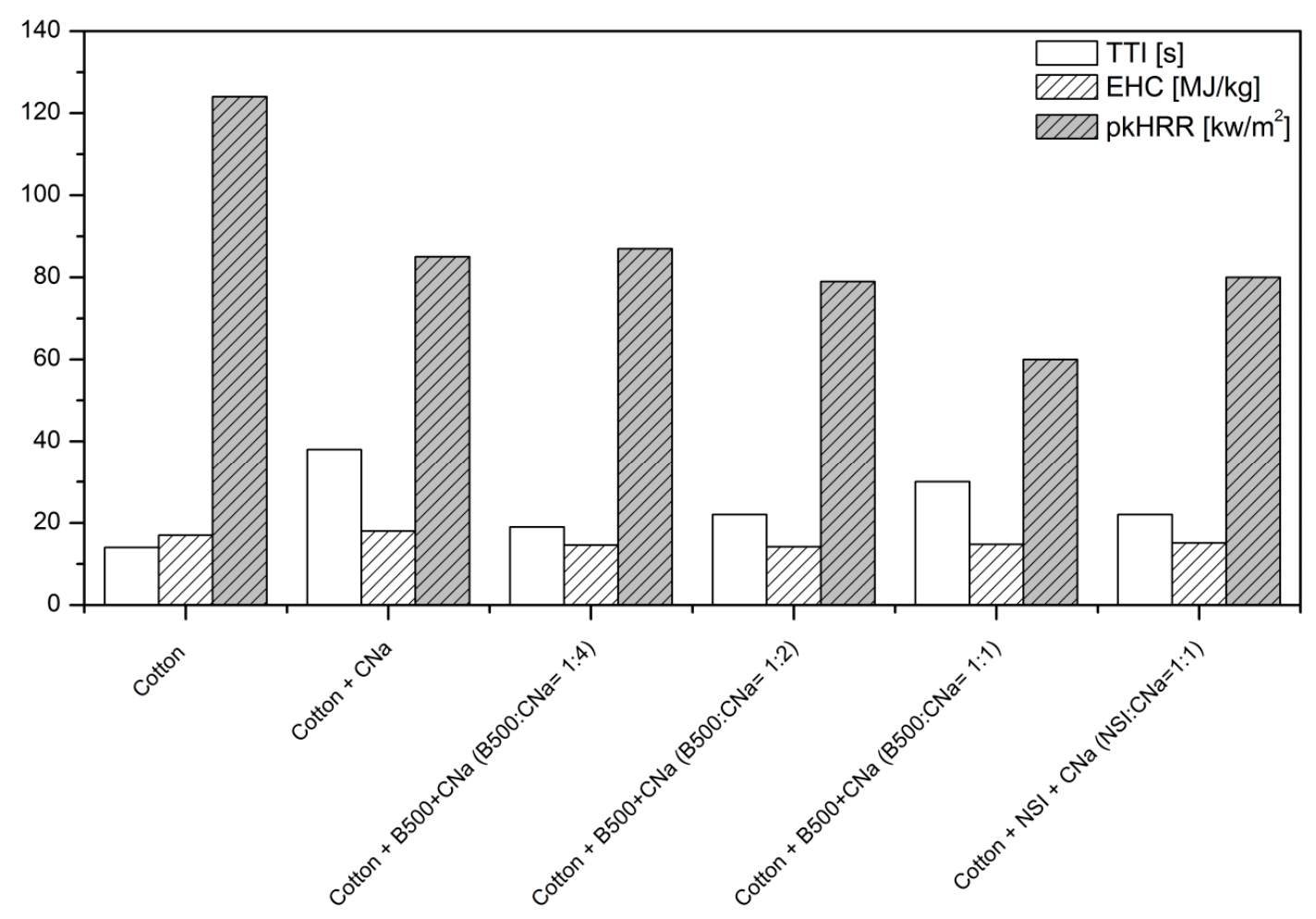

Figure 2. TTI, EHC and pkHRR values for cotton treated with $\mathrm{CNa}$ and $\mathrm{B} 500$ chemical. 
An alternative approach to increasing the nanoparticle amount deposited on the cotton surface has been the use of a binder able to covalently link the hydroxyl groups on $\mathrm{CNa}$ surface to the hydroxyl group of $\mathrm{C}(6)$ glucose unit present in the cotton cellulose structure. With this aim, dicyanamide-formaldehyde polymer, melamine-formaldehyde and dimethylol(dihydroxy)ethyleneurea (C, D and E species in Table 3) were employed to link different nominal amounts of sodium cloisite (namely, 1, 2 and $5 \mathrm{wt} \%$ ). From the collected data (Table 6), it is possible to conclude that all the chosen binders significantly affects cotton combustion, increasing TTI and decreasing pkHRR, regardless of nanoparticle amount. Also the smoke release has been reduced in the presence of these binders as compared with the cotton $+\mathrm{CNa}$ sample (TSR values).

Since sodium cloisite has shown some level of flame-retardant performances for both the polyester and cotton, two different blends of them were treated in a similar manner with the clay. Tables 8 and 9 show that $\mathrm{CNa}$ is an efficient flame retardant for PET:cotton $=85: 15$ and PET:cotton $=65: 35$ blends since its presence increases their TTI (52 s vs. $35 \mathrm{~s}$ and $11 v s .24 \mathrm{~s}$, respectively) and slightly decreases pkHRR (150 kW/m² vs. $107 \mathrm{~kW} / \mathrm{m}^{2}$ and $140 \mathrm{~kW} / \mathrm{m}^{2} v s .125 \mathrm{~kW} / \mathrm{m}^{2}$, respectively), regardless of blend composition. However, once again, the presence of $\mathrm{CNa}$ causes a strong increase of smoke generation during combustion.

\subsubsection{Hydrotalcites}

As it is well known [24], hydrotalcites are anionic nanoclays with a chemical formula of $\mathrm{Mg}_{6} \mathrm{Al}_{2}\left(\mathrm{CO}_{3}\right)\left(\mathrm{OH}_{16}\right) \cdot 4\left(\mathrm{H}_{2} \mathrm{O}\right)$ (Table 1). The high water content present in the structure of HT (hydrotalcite) can be responsible for a delay in TTI, as already demonstrated by our research group for both the polyester [9] (Figure 1) and cotton fabrics [23]. Indeed, these nanoparticles are able to partially protect a polymer from the heat and oxygen as they form a ceramic layer during combustion, releasing a great amount of water upon heating. In this manner, the degradation products released by the polymer are diluted, causing a significant delay of ignition. This has been observed on both synthetic (namely, PET) and natural (cotton) fabrics. In detail, comparing the data collected in Table 4, it is possible to observe that PET TTI increases from 166 up to $226 \mathrm{~s}$ and pkHRR decreases from 90 to $56 \mathrm{~kW} / \mathrm{m}^{2}$ thanks to the presence of adsorbed hydrotalcite (Figure 1). The use of a binder-like PES (Table 3) further promotes TTI increase (244 s and $226 \mathrm{~s} v s .160 \mathrm{~s}$ for PET + PES + HT, PET + HT and PET, respectively). In the meantime, these nanoparticles significantly affect also the combustion kinetics as shown by comparing respective sample EHC and pkHRR values in Table 4 and plotted in Figure 1. From an overall consideration, the highest FPI value registered is for the PET + PES + HT system in comparison with neat PET and the other lamellar-based systems under investigation $\left(4.36 \mathrm{sm}^{2} / \mathrm{kW}\right.$ vs. $1.89 \mathrm{sm}^{2} / \mathrm{kW}$, Table 4$)$.

The same increasing trend in TTI has been observed using the polyester with the higher area density (148 s vs. 106 s for PET + HT and PET, respectively, Table 5). The use of different dispersing agents, however, did not suggest their use as means to further increase this parameter.

Referring to cotton, Table 10 reports the collected data of cotton treated with HT for two impregnation times (namely, 30 and $60 \mathrm{~min}$ ): it is clear that this nanoparticle acts by the same way already observed for PET, by improving cotton TTI and significantly reducing pkHRR; as a consequence, the FPI value of HT-treated fabrics is higher than that of untreated cotton [22]. Comparing the two samples obtained 
with different times, it is possible to note that a more prolonged immersion time does not increase the nanoparticle effect and probable amount, and thus the resulting flame-retardant properties.

Table 10. Combustion data of untreated and hydrotalcite-treated cotton $\left(d=210 \mathrm{~g} / \mathrm{m}^{2}\right)$ by cone calorimetry [22].

\begin{tabular}{cccc}
\hline Sample & TTI (s) & pkHRR $\left(\mathbf{k W} / \mathbf{m}^{\mathbf{2}}\right)$ & FPI $\left(\mathbf{s m}^{\mathbf{2}} / \mathbf{k W}\right)$ \\
\hline Cotton & $14 \pm 1$ & $124 \pm 6$ & $0.11 \pm 0.02$ \\
Cotton + HT (30 min) & $34 \pm 1$ & $87 \pm 1$ & $0.39 \pm 0.06$ \\
Cotton + HT (60 min) & $22 \pm 5$ & $94 \pm 2$ & $0.23 \pm 0.03$ \\
\hline
\end{tabular}

As much as hydrotalcite has improved fire performance of polyester [9] and cotton [22], analogous experiments and results for PET:cotton $=65: 35$ blend show that the TTI increases from 11 to $74 \mathrm{~s}$ (Table 9).

\subsubsection{Bohemites}

As far as bohemites are concerned, theoretically these nanoparticles have intrinsic flame-retardant features as they are aluminum oxide hydroxides, the $\gamma-\mathrm{AlO}(\mathrm{OH})$, that dehydrate in the range of 100-300 ${ }^{\circ} \mathrm{C}$, releasing water and subsequently transforming into crystalline the $\gamma-\mathrm{Al}_{2} \mathrm{O}_{3}$ phase at circa $420{ }^{\circ} \mathrm{C}$ [25]. In doing so, during the first degradation step, the volatile products generated by polymers under irradiation are diluted and the ignition is reached after a longer time as compared with pure polymeric material. Furthermore, the ceramic barrier due to the presence of the just-formed alumina inhibits the further combustion. Table 11 shows that p-toluenesulfonate salt bohemite (OS1) is able to delay cotton TTI (alone or covalently linked with some binders); furthermore, its pkHRR is significantly reduced, as are the production of smoke and $\mathrm{CO}$ and $\mathrm{CO}_{2}$ yields [23]. All formulations under investigations have exhibited a higher FPI value if compared with untreated cotton.

Table 11. Combustion data of untreated and bohemite-treated cotton $\left(d=210 \mathrm{~g} / \mathrm{m}^{2}\right)$ by cone calorimetry [23].

\begin{tabular}{cccc}
\hline Sample & TTI (s) & pkHHR $\left(\mathbf{k W} / \mathbf{m}^{2} \mathbf{g}\right)$ & FPI $\left(\mathbf{s m}^{2} \mathbf{g} / \mathbf{k W}\right)$ \\
\hline Cotton & $14 \pm 1.0$ & $57 \pm 6$ & $0.24 \pm 0.02$ \\
Cotton + OS1 & $22 \pm 2.2$ & $50 \pm 5$ & $0.44 \pm 0.04$ \\
Cotton + A + OS1 & $22 \pm 2.0$ & $41 \pm 4$ & $0.54 \pm 0.05$ \\
Cotton + B + OS1 & $20 \pm 2.0$ & $45 \pm 4$ & $0.44 \pm 0.0$ \\
Cotton + C + OS1 & $20 \pm 2.0$ & $36 \pm 3$ & $0.56 \pm 0.06$ \\
Cotton + D + OS1 & $21 \pm 1.5$ & $35 \pm 5$ & $0.60 \pm 0.06$ \\
Cotton + E + OS1 & $20 \pm 1.0$ & $46 \pm 4$ & $0.43 \pm 0.04$ \\
\hline
\end{tabular}

As far as polyester is concerned, bohemite alone does not affect its TTI; however, it reduces the EHC (15.0 MJ/kg vs. $15.8 \mathrm{MJ} / \mathrm{kg}$ for PET + OS1 and PET, respectively, Table 4), pkHRR (from 90 to $70 \mathrm{~kW} / \mathrm{m}^{2}$ ) (Figure 1), TSR (from 90 to $76 \mathrm{~m}^{2} / \mathrm{m}^{2}$ ), SEA (from 610 to $394 \mathrm{~m}^{2} / \mathrm{kg}$ ) and $\mathrm{CO}$ and $\mathrm{CO}_{2}$ yields. The use of a binder, such as PES, can be useful if the main goal is to increase the TTI (from 160 up to $258 \mathrm{~s}$, Table 4, Figure 1), but it does not decrease the combustion kinetics; indeed, the pkHRR is 
nearly constant. As a consequence, PET + OS1 exhibited a lower FPI value with respect to PET while for PET + PES + OS1 the opposite trend was observed (Table 4).

\subsection{Globular Nanoparticles}

Globular nanoparticles such as titania (anatase crystalline form), silica and octapropylammonium POSS have been deposited on polyester [9] and cotton [22]. In the former case, apart from a slight reduction of pkHRR (Figure 1), it is important to highlight their effect on smoke release: in particular, these nanoparticles significantly reduce $\mathrm{CO}$ and $\mathrm{CO}_{2}$ yields (Table 4). This trend has been also found when polyesters with a higher area density and denser structure are treated by silica (Table 5). However, silica does not provide the same results in terms of FPI which remains identical to that of neat polyester. The amount of nanoparticle present on the surface is likely very low and thus two dispersing agents (namely, NSI and WP, Table 3) were employed in order to increase the nanoparticle amount in suspension and consequently be available to impregnate fabrics. Indeed, comparing the TTI values reported in Table 5, a significant increase depending on time immersion has been observed. Once again, the use of such agents has, however, caused an increase of pkHRR, already observed for hydrotalcite.

As far as cotton is concerned, silica turned out to be efficient in reducing its pkHRR, as is well shown by the collected data reported in Table 12.

Negligible effects were found when silica was used for fire-protecting the blends under investigation, as demonstrated by the data reported in Tables 8 and 9 .

Table 12. Combustion data of untreated and silica-treated cotton $\left(d=210 \mathrm{~g} / \mathrm{m}^{2}\right)$ by cone calorimetry [22].

\begin{tabular}{cccc}
\hline Sample & TTI (s) & pkHRR $\left(\mathbf{k W} / \mathbf{m}^{2}\right)$ & FPI $\left(\mathbf{s m}^{2} / \mathbf{k W}\right)$ \\
\hline Cotton & $14 \pm 1$ & $124 \pm 6$ & $0.11 \pm 0.02$ \\
Cotton $+\mathrm{SiO}_{2}(30 \mathrm{~min})$ & $17 \pm 6$ & $99 \pm 3$ & $0.17 \pm 0.02$ \\
Cotton $+\mathrm{SiO}_{2}(60 \mathrm{~min})$ & $20 \pm 8$ & $95 \pm 3$ & $0.21 \pm 0.02$ \\
\hline
\end{tabular}

Since the silica performance may be affected by its poor dispersion level in water, the authors have supposed that a silicon-based structure soluble in water, such as octapropylammonium POSS, could be suitable in order to create a silicon-based barrier for the fabrics. Table 6 and Figure 3 show that this molecule is able to impart significant enhancement of the flame-retardant properties relative to cotton, in particular when a binder such as melamine-formaldehyde (D) is employed [23]. The highest TTI, the lowest pkHRR and EHC have been found with a POSS nominal concentration of $5 \mathrm{wt} \%$.

As far as polyester is concerned, Table 4 shows that such POSS can be considered a good flame retardant, able to increase the TTI and to decrease pkHRR (Figure 1), $\mathrm{CO}$ and $\mathrm{CO}_{2}$ production. Among the globular nanoparticles, POSS seems to be the most promising. 


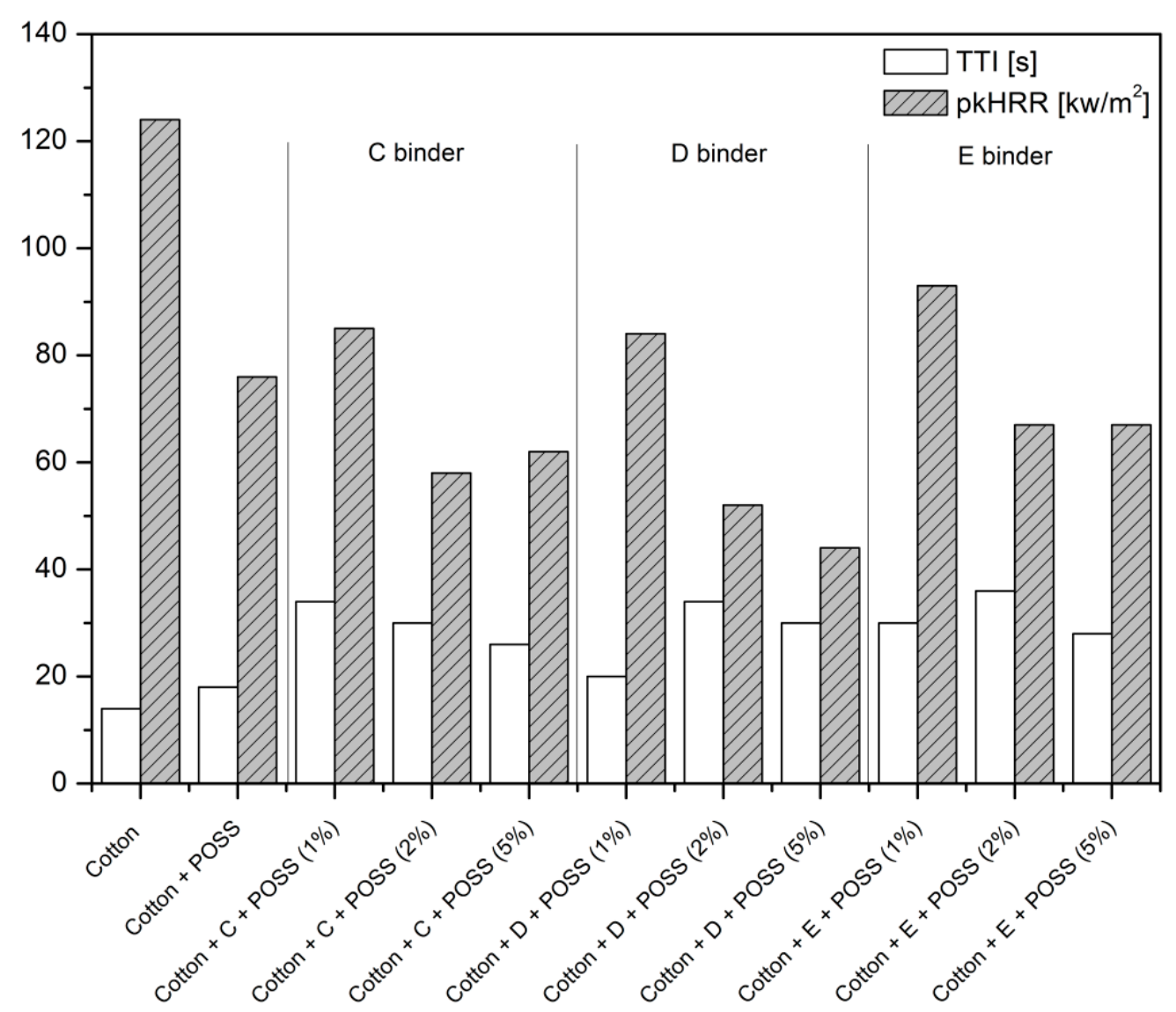

Figure 3. TTI and pkHRR values of cotton treated with C, D or E binders and POSS.

\subsection{Combination of Lamellar and Globular Nanoparticles}

The results discussed above have pointed out that the low amounts of nanoparticles deposited on the fiber surfaces are not able to completely and homogeneously cover the fabric surface and, thus, the nanoparticles do not form an efficient physical barrier capable of protecting the substrate from fire.

Previous work has already demonstrated that the combination of lamellar and globular nanoparticles can be a useful means of reaching the optimal performances for enhancing the flame retardancy of polyester [9] and cotton [22]. In particular, the combination of a lamellar nanoparticle as hydrotalcite and a globular one such as silica appeared to be very efficient in increasing the TTI of polyester in a dramatic way (Figure 1); in addition, further analyses have shown that this system is able to reduce the production of $\mathrm{CO}$ and $\mathrm{CO}_{2}$ in a remarkable way (Table 4).

Similarly interesting results have been collected for cotton (Table 6) and for the PET:cotton $=65: 35$ blend (Table 9), as well.

An alternative combination for cotton consisting of $\mathrm{CNa}$ and POSS has exhibited remarkable results (Table 6), while varying the amount of nanoparticles or using a binder. More specifically, CNa was covalently linked to $\mathrm{C}(6)$ of cellulose using $\mathrm{C}$, D or E binders (Table 3) and subsequently such fabrics were treated with a POSS water solution. The results in Table 6 show, from a general perspective, that this system turned out to be efficient for enhancing cotton's fire stability: all the parameters are improved as compared with those of pure cotton. However, the fabrics treated by only POSS have 
shown the highest improvements in fire performance, as evidenced by comparing all FPI values reported in Table 6 (when the amount of employed nanoparticles is fixed) and plotted in Figure 4.

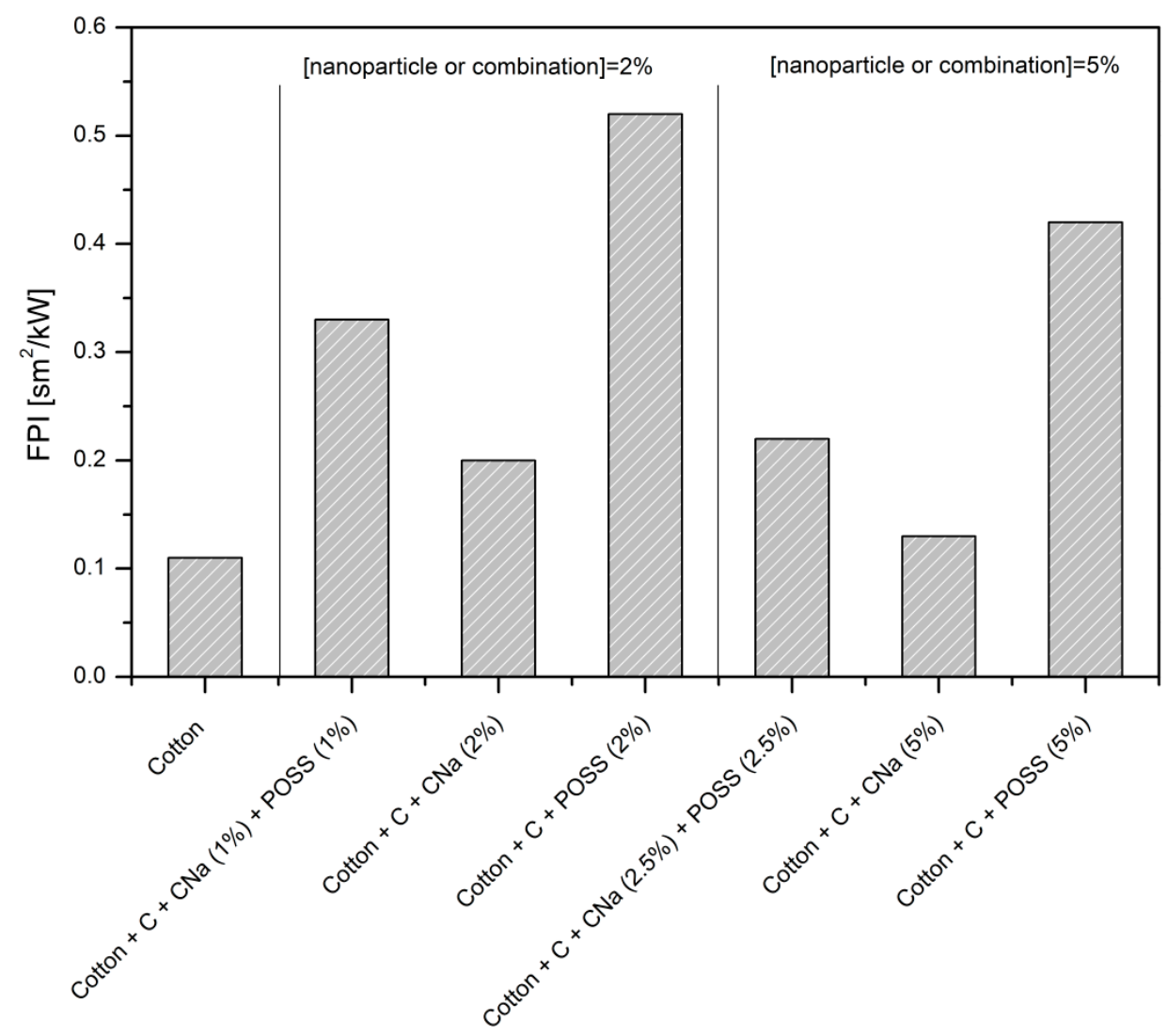

Figure 4. FPI values of cotton treated with $\mathrm{C} / \mathrm{CNa}$ or $\mathrm{C} / \mathrm{POSS}$ pairs or their combinations.

\subsection{Combination of a Commercial Phosphorus-Based Flame Retardant with POSS}

For a possible industrial application, all the formulations studied by cone calorimetry have been tested following the ISO15025 standard that is a specific vertical fabric strip test for protective garments (data not given here). From an overall consideration, POSS turned out to be the most promising nanoparticle to enhance polyester flame retardancy. Indeed, these specimens do not burn when a methane flame was applied directly on a fabric surface. Unfortunately, the same trend has not been observed for cotton and the PET:cotton $=65: 35$ blend. For this reason, it was thought that a possible combination of a phosphorus-based flame retardant (namely, Pyrovatex ${ }^{\circledR}$-like [13]) could be combined with POSS in order to enhance the overall flame retardancy level of such blends. Figure 5 shows the collected results of PET:cotton $=65: 35$ treated with this flame retardant at $10 \mathrm{wt} \%$ (the standard concentration commonly employed being 13\%-19\%) and different POSS amounts (namely, 5, 15 and $30 \mathrm{wt} \%$ ) by cone calorimetry. By increasing the POSS amount, TTI increases and pkHRR decreases in a remarkable way and, in addition, the most interesting results are shown in Figure 6, where the residues of tested specimens after combustion are reported. The final residue of the untreated blend is almost completely destroyed after the tests, whereas that of fabric treated by only a phosphorus-flame retardant consists of a thin film; on the contrary, this residue becomes more coherent and compact by adding POSS. Its consistency appears to be dependent on nanoparticle amount. 


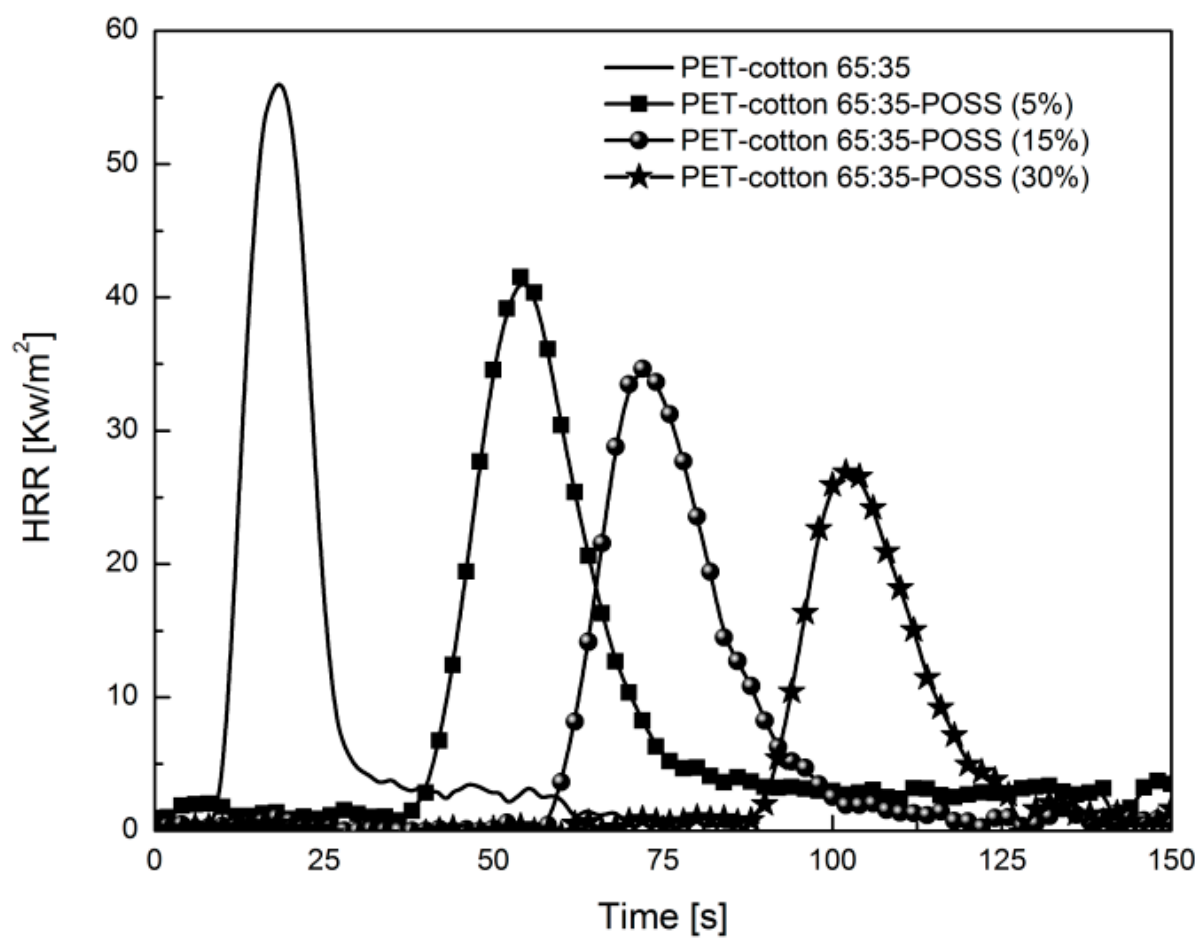

Figure 5. HRR curves of neat PET:cotton blend and of fabrics treated with a phosphorus-based flame retardant with different POSS amounts.

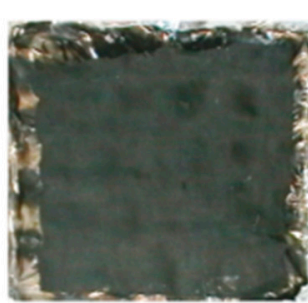

Residue of the blend treated with P-based flame retardant

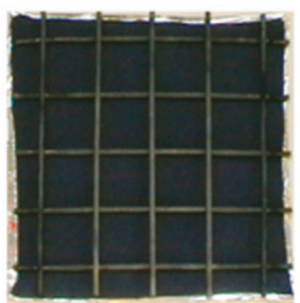

Blend fabric

$\Lambda, \mathrm{O}_{2}$
$\Lambda, \mathrm{O}_{2}$

$$
\mathrm{O}_{2}, \Delta
$$

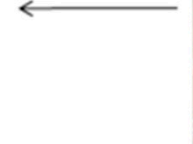

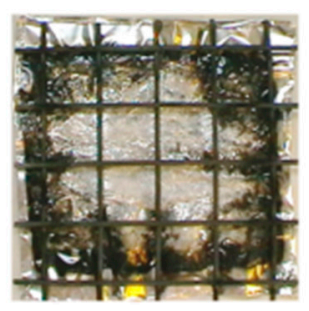

Residue of the neat blend

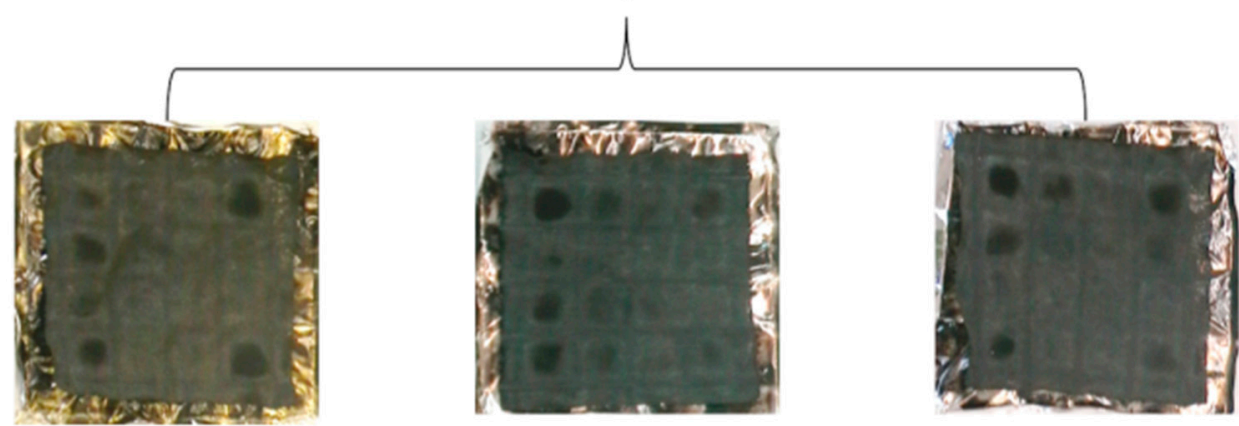

Residue of the blend treated with P-based flame retardant + POSS

(5wt.-\% POSS 15 wt. $\%$ POSS

30wt.-\% POSS )

Figure 6. Residues of untreated PET:cotton blend and fabrics treated with a phosphorus-based flame retardant and different POSS amounts after cone calorimetry tests. 


\section{Conclusions}

In the present work, the use of a wide set of nanoparticle types and their combinations has been reviewed in order to establish the most efficient system able to enhance the flame retardancy of polyester, cotton and two of their blends. All the formulations taken into consideration have been studied from the combustion point of view, by using an oxygen-consuming cone calorimeter at $35 \mathrm{~kW} / \mathrm{m}^{2}$.

On the basis of the aforementioned results and, in particular, of the calculated FPI values, it is possible to establish a form of ranking of nanoparticles (from the least to the most efficient), determined by the protective role exerted on the fabric resistance to combustion:

(i) Polyester $\left(d=171 \mathrm{~g} / \mathrm{m}^{2}\right)\left(\mathrm{FPI}=1.89 \mathrm{sm}^{2} / \mathrm{kW}\right)$ :

Based on an increasing value for fire performance index (FPI):

$\mathrm{SiO}_{2}<\mathrm{OS} 1<\mathrm{POSS}<\mathrm{TiO}_{2}<\mathrm{CNa}<\mathrm{HT}+\mathrm{SiO}_{2}(30+30 \mathrm{~min})<\mathrm{HT}<\mathrm{HT}+\mathrm{SiO}_{2}(60+60$ min $)$ (Figure 7);

$\mathrm{SiO}_{2}\left(1.30 \mathrm{sm}^{2} / \mathrm{kW}\right)<\mathrm{OS} 1\left(1.50 \mathrm{sm}^{2} / \mathrm{kW}\right)<\operatorname{POSS}\left(1.97 \mathrm{sm}^{2} / \mathrm{kW}\right)<\mathrm{TiO}_{2}\left(2.17 \mathrm{sm}^{2} / \mathrm{kW}\right)<\mathrm{CNa}$ $\left(2.35 \mathrm{sm}^{2} / \mathrm{kW}\right)<\mathrm{HT}+\mathrm{SiO}_{2}(30+30 \mathrm{~min})\left(2.82 \mathrm{sm}^{2} / \mathrm{kW}\right)<\mathrm{HT}\left(2.97 \mathrm{sm}^{2} / \mathrm{kW}\right)<\mathrm{HT}+\mathrm{SiO}_{2}$ $(60+60 \mathrm{~min})\left(3.38 \mathrm{sm}^{2} / \mathrm{kW}\right)$.

From this list and taking into account the unmodified PET, it is possible to conclude that one single type of nanoparticle is not able to confer optimal performances to polyester: indeed, the hydrotalcite-silica combination turned out to be the most efficient flame-retardant system.

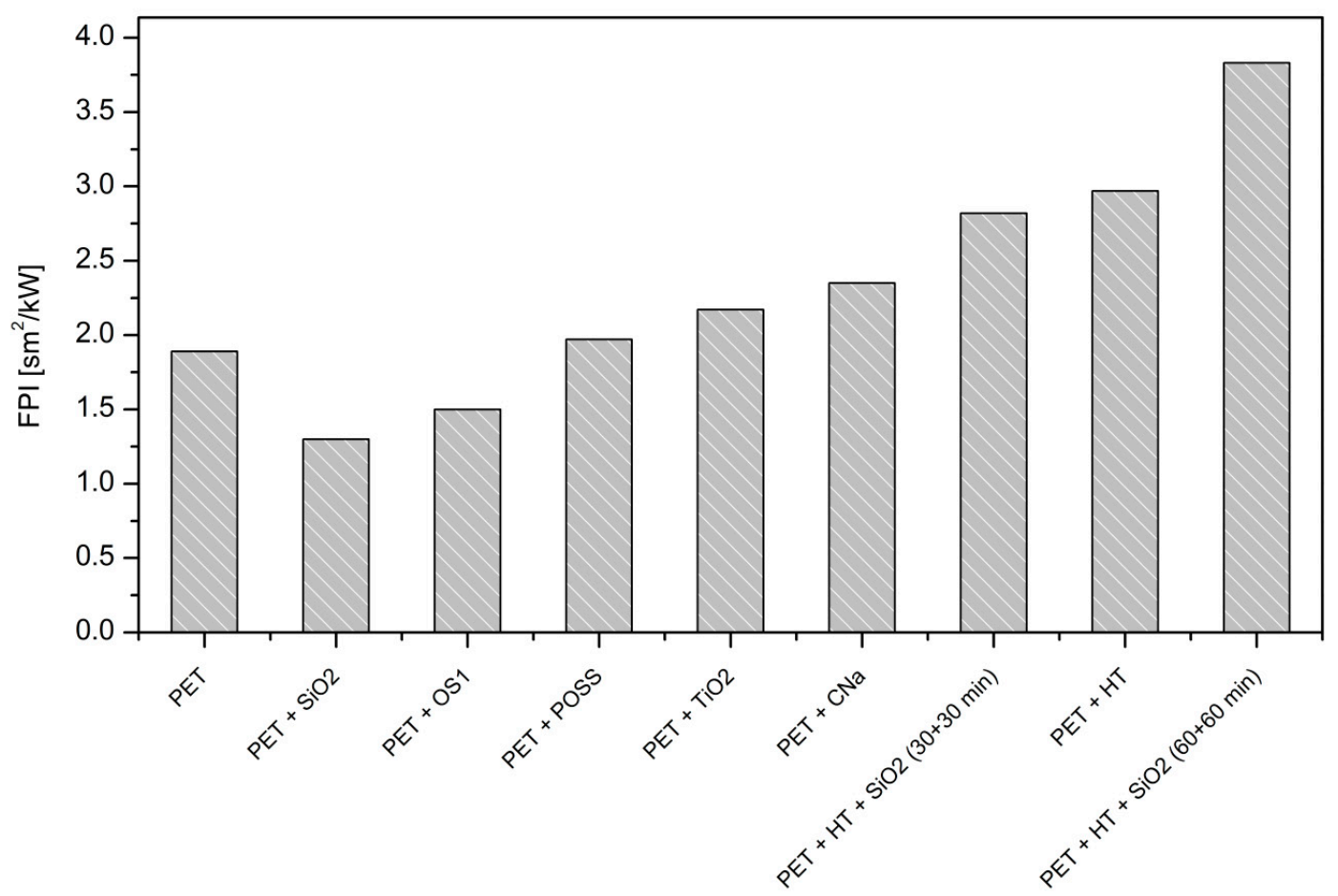

Figure 7. FPI values for nanoparticle-treated PET (area density $=171 \mathrm{~g} / \mathrm{m}^{2}$ ).

(ii) For the blend having the highest PET amount (namely, PET:cotton $=85: 15$ ), CNa has shown the best performance in terms of FPI $\left(0.49 \mathrm{sm}^{2} / \mathrm{kW} v s .0 .24 \mathrm{sm}^{2} / \mathrm{kW}\right.$ for unmodified blend); this nanoparticle has also shown itself to be efficient in the case of the blend having the lowest 
PET amount (namely, PET:cotton =65:35); in the latter case, HT, silica and the HT-silica combination have imparted improved fire performances to this blend, as well.

(iii) Polyester $\left(d=490 \mathrm{~g} / \mathrm{m}^{2}\right)$ :

In the case of the higher area density polyester, only a slight increase of its flame-retardancy performance employing sodium cloisite and hydrotalcite has been observed (FPI $=0.64$ and 0.62 respectively $v s .0 .50 \mathrm{sm}^{2} / \mathrm{kW}$ for unmodified fabric).

(iv) Cotton (FPI $\left.=0.11 \mathrm{sm}^{2} / \mathrm{kW}\right)$ :

Based on an increasing value for fire performance index (FPI), the next ranking follows:

$\mathrm{SiO}_{2}(30 \mathrm{~min})<\mathrm{SiO}_{2}(60 \mathrm{~min})<\mathrm{POSS}<\mathrm{HT}+\mathrm{SiO}_{2}(30+30 \mathrm{~min})<\mathrm{HT}<\mathrm{HT}+\mathrm{SiO}_{2}$ $(60+60 \mathrm{~min}) \approx \mathrm{OS} 1 \approx \mathrm{CNa}$ based on the following FPI values (Figure 8);

$\mathrm{SiO}_{2}(30 \mathrm{~min})\left(0.17 \mathrm{sm}^{2} / \mathrm{kW}\right)<\mathrm{SiO}_{2}(60 \mathrm{~min})\left(0.21 \mathrm{sm}^{2} / \mathrm{kW}\right)<\operatorname{POSS}\left(0.24 \mathrm{sm}^{2 / k W}\right)<\mathrm{HT}+\mathrm{SiO}_{2}$ $(30+30 \mathrm{~min})\left(0.32 \mathrm{sm}^{2} / \mathrm{kW}\right)<\mathrm{HT}\left(0.39 \mathrm{sm}^{2} / \mathrm{kW}\right)<\mathrm{HT}+\mathrm{SiO}_{2}(60+60 \mathrm{~min})\left(0.43 \mathrm{sm}^{2} / \mathrm{kW}\right) \approx \mathrm{OS} 1$ $\left(0.44 \mathrm{sm}^{2} / \mathrm{kW}\right) \approx \mathrm{CNa}\left(0.45 \mathrm{sm}^{2} / \mathrm{kW}\right)$.

All the nanoparticles under study show similar behavior in that they improve cotton fire performance to varying degrees.

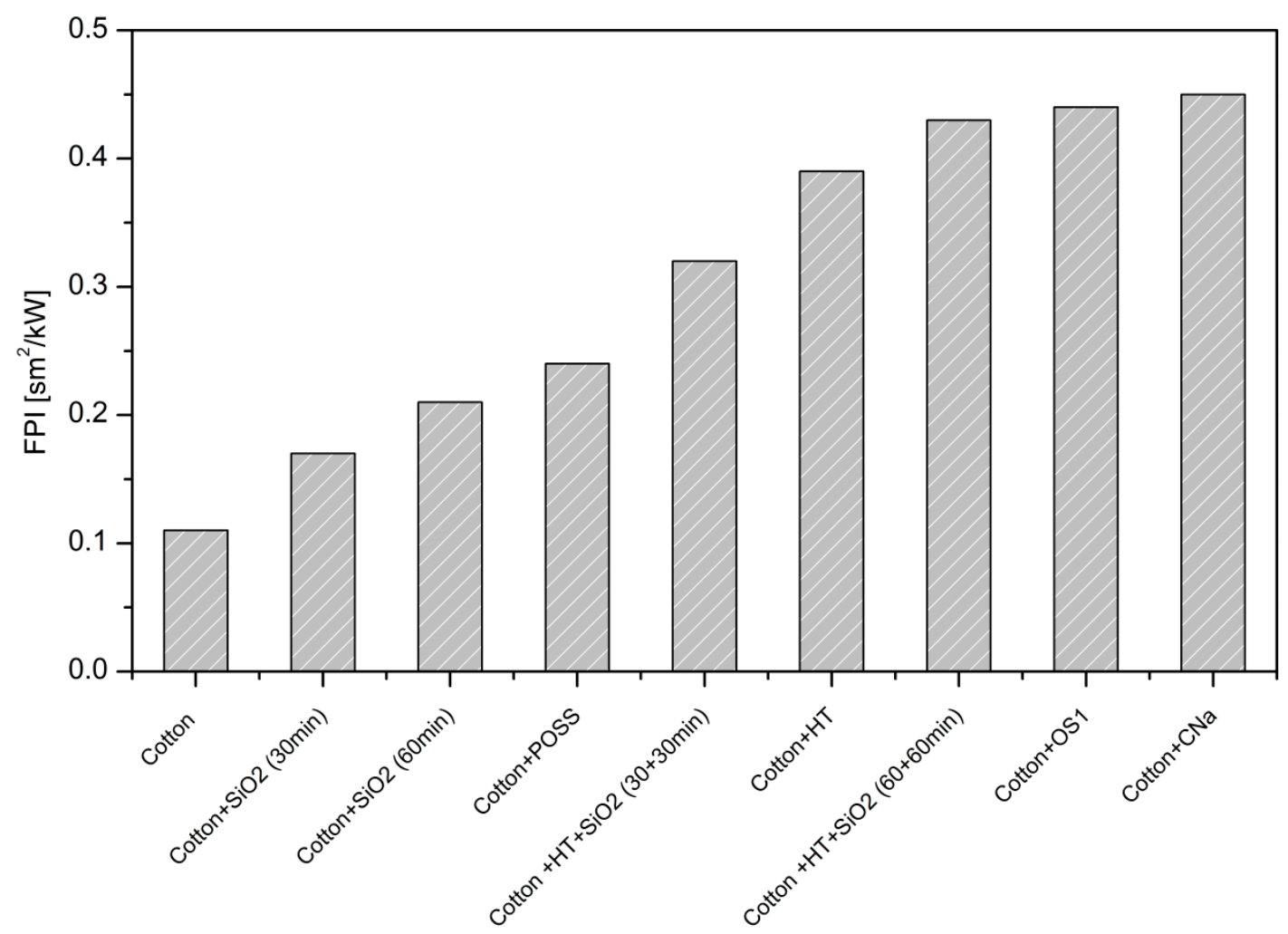

Figure 8. FPI values for nanoparticle-treated cotton.

\section{Acknowledgments}

The authors would like to thank the European Commission for the economical funding of FRONT (Flame Retardant on Textiles) project 2008/2010 (7th Framework Program, contract No. 222486), Alessandro Scalvedi and Fabio Cuttica for the technical support. 


\section{Conflicts of Interest}

The authors declare no conflict of interest.

\section{References}

1. Brushlinsky, N.N.; Sokolov, S.V.; Wagner, P.; Hall, J.R. World Fire Statistics. Report No. 10; Centre of Fire Statistics, International Association of Fire and Rescue Service: Geneva, Switzerland, 2006.

2. Kobes, M.; Groenewegen, K. Consumer Fire: European Statistics and Potential Fire Safety Measures; Austrian Federal Ministry Labor, Social Affairs and Consumer Protection: Wien, Austria, 2009.

3. Babrauskas, V. Upholstered furniture heat release rRates: Measurements and estimation. J. Fire Sci. 1983, 1, 9-32.

4. Babrauskas, V. The cone calorimeter. In Heat Release in Fires; Babrauskas, V., Grayson, S.J., Eds.; Chapman \& Hall: London, UK, 1995; pp. 61-91.

5. Babrauskas, V.; Baroudi, D.; Myllymaki, J.; Kokkala, M. The cone calorimeter used for predictions of the full-scale burning behaviour of upholstered furniture. Fire Mater. 1997, 21, 95-105.

6. Standard Test Method for Determining the Heat Release Rate of Upholstered Furniture and Mattress Components or Composites Using a Bench Scale Oxygen Consumption Calorimeter 2004; ASTM E1474; ASTM International: West Conshohocken, PA, USA, 2004.

7. Standard Test Method for Determining the Heat Release Rate and Other Fire-Test-Response Characteristics of Wallcovering Composites Using a Cone Calorimeter 2002; ASTM E1740; ASTM International: West Conshohocken, PA, USA, 2002.

8. Tata, J.; Alongi, J.; Carosio, F.; Frache, A. Optimization of the procedure to burn textile fabrics by cone calorimeter: Part I. Combustion behavior of polyester. Fire Mater. 2011, 35, 397-409.

9. Tata, J.; Alongi, J.; Frache A. Optimization of the procedure to burn textile fabrics by cone calorimeter: Part II. Results and discussion on nanoparticle finished polyester. Fire Mater. 2012, 36, 527-536.

10. Plastics-Smoke Generation-Part 1: Guidance on Optical-Density Testing $1996+$ Part 2: Determination of Optical Density by a Single-Chamber Test 2002; ISO 5659; International Organization for Standardization: Geneva, Switzerland.

11. Fire Test, Reaction to Fire-Part 1: Rate of Heat Release (Cone Calorimeter Method) + Part 2: Smoke Production Rate (Dynamic Measurement) + Part 3: Guidance on Measurement 2002 and 2003; ISO 5660; International Organization for Standardization: Geneva, Switzerland.

12. Reaction to Fire Tests for Floorings-Part 1: Determination of the Burning Behaviour Using a Radiant Heat Source + Part 2: Determination of Flame Spread at a Heat Flux Level of $25 \mathrm{~kW} / \mathrm{m}^{2}$ 2002; ISO 9239; International Organization for Standardization: Geneva, Switzerland.

13. Horrocks, A.R. Flame retardant challenges for textiles and fibres: New chemistry versus innovatory solutions. Polym. Degrad. Stab. 2011, 96, 377-392. 
14. Alongi, J.; Carosio, F.; Horrocks, A.R.; Malucelli, G. Update on Flame Retardant Textiles: State of the Art, Environmental Issues and Innovative Solutions; Smithers RAPRA Publishing: Shawbury, UK, 2013.

15. Van der Veen, I.; De Boer, J. Phosphorus flame retardants: Properties, production, environmental occurrence, toxicity and analysis. Chemosphere 2012, 88, 1119-1153.

16. Alongi, J.; Frache, A.; Malucelli, G.; Camino, G. Multi-component flame retardant coatings. In Handbook of Fire Resistant Textiles; Selcen Kilinc, F., Ed.; Woodhead Publishing: Cambridge, UK, 2013; Chapter 4, pp. 68-93.

17. Alongi, J.; Carosio, F.; Malucelli, G. Current emerging techniques to impart flame retardancy to fabrics. Polym. Degrad. Stab. 2014, 106, 138-149.

18. Malucelli, G.; Carosio, F.; Alongi, J.; Fina, A.; Frache, A.; Camino, G. Materials engineering for surface-confined flame retardancy. Mater. Sci. Eng. R Rep. 2014, 84, 1-20.

19. Alongi, J.; Malucelli, G. State of the art and perspectives on sol-gel derived hybrid architectures for flame retardancy of textiles. J. Mater. Chem. 2012, 22, 21805-21809.

20. Schartel, B.; Bartholmai, M.; Knoll, U. Some comments on the main fire retardancy mechanisms in polymer nanocomposites. Polym. Adv. Technol. 2006, 17, 772-776.

21. Carosio, F.; Alongi, J.; Frache, A. Influence of surface activation by plasma and nanoparticle adsorption on the morphology, thermal stability and combustion behavior of PET fabrics. Eur. Polym. J. 2011, 47, 893-902.

22. Alongi, J.; Tata, J.; Frache, A. Hydrotalcite and nanometric silica as finishing additives to enhance the thermal stability and flame retardancy of cotton. Cellulose 2011, 18, 179-190.

23. Alongi, J.; Brancatelli, G.; Rosace, G. Thermal properties and combustion behavior of POSS- and bohemite-finished cotton fabrics. J. Appl. Polym. Sci. 2012, 123, 426-436.

24. Costantino, U.; Nocchetti, M.; Sisani, M.; Vivani, R. Recent progress in the synthesis and application of organically modified hydrotalcites. Z. Krist. 2009, 224, 273-281.

25. Esposito Corcione, C.; Frigione, M.; Maffezzoli, A.; Malucelli, G. Photo-DSC and real time-FT-IR kinetic study of a UV curable epoxy resin containing o-Boehmites. Eur. Polym. J. 2008, 44, 2010-2023.

(C) 2014 by the authors; licensee MDPI, Basel, Switzerland. This article is an open access article distributed under the terms and conditions of the Creative Commons Attribution license (http://creativecommons.org/licenses/by/4.0/). 